\title{
Allogeneic Venous Grafts of Different Origin Used for Portal Vein Reconstruction After Pancreaticoduodenectomy - Experimental Study
}

\author{
RICHARD PALEK ${ }^{1,2}$, ALENA JONASOVA ${ }^{3}$, JACHYM ROSENDORF ${ }^{1,2}$, PATRIK MIK $^{2,4}$, \\ KRISTYNA BAJCUROVA ${ }^{2,5}$, PETR HOSEK ${ }^{2}$, VLADIMIRA MOULISOVA ${ }^{2}$, LADA EBERLOVA $^{2,4}$, \\ LENKA HAIDINGEROVA ${ }^{6}$, ONDREJ BRZON ${ }^{2}$, LUKAS BEDNAR ${ }^{2}$, TOMAS KRIZ ${ }^{2}$, \\ MARTIN DOLANSKY ${ }^{2}$, VLADISLAV TRESKA ${ }^{1}$, ZBYNEK TONAR $^{2,7}$, JAN VIMMR $^{3}$ and VACLAV LISKA ${ }^{1,2}$ \\ ${ }^{1}$ Department of Surgery, Faculty of Medicine in Pilsen, Charles University, Pilsen, Czech Republic; \\ ${ }^{2}$ Biomedical Center, Faculty of Medicine in Pilsen, Charles University, Pilsen, Czech Republic; \\ ${ }^{3}$ Department of Mechanics, Faculty of Applied Sciences, University of West Bohemia, Pilsen, Czech Republic; \\ ${ }^{4}$ Department of Anatomy, Faculty of Medicine in Pilsen, Charles University, Pilsen, Czech Republic; \\ ${ }^{5}$ Department of Medical Imaging, Faculty of Medicine in Pilsen, Charles University, Pilsen, Czech Republic; \\ ${ }^{6}$ Department of Anesthesiology and Resuscitation, \\ Faculty of Medicine in Pilsen, Charles University, Pilsen, Czech Republic; \\ ${ }^{7}$ Department of Histology and Embryology, \\ Faculty of Medicine in Pilsen, Charles University, Pilsen, Czech Republic
}

\begin{abstract}
Background: In clinical medicine, little is known about the use of allografts for portal vein $(P V)$ reconstruction after pancreaticoduodenectomy (PD). Portal and caval systems are physiologically different, therefore the properties of allografts from caval and portal systems were studied here in a pig model. Materials and Methods: PD with $P V$ reconstruction with allogeneic venous graft from $P V$ or inferior vena cava (IVC) was performed in 26 pigs. Biochemical analysis and ultrasonography measurements were performed during a 4-week monitoring period. Computer simulations were used to evaluate haemodynamics in reconstructed $P V$ and explanted allografts were histologically examined. Results: The native PV and IVC grafts varied in histological structure but were able to adapt morphologically after transplantation. Computer simulation suggested PV grafts to be more susceptible to thrombosis development. Thrombosis of reconstructed PV occurred in four out of five cases in PV group. Conclusion: This study
\end{abstract}

Correspondence to: Richard Palek, Alej Svobdy 76, 32300 Pilsen, Czech Republic. Tel: +420 722435599, e-mail: palekrichard@ gmail.com; Václav Liška, Alej Svobdy 76, 32300 Pilsen, Czech Republic. Tel: +420 732160287, e-mail: vena.liska@skaut.cz

Key Words: Pancreaticoduodenectomy, portal vein reconstruction, allogeneic grafts, portal vein haemodynamics, computer blood flow simulation. supports the use of allografts from caval system for $P V$ reconstruction in clinical medicine when needed.

Pancreatic cancer is one of the leading causes of cancer mortality in developed countries; its incidence has been rising over last decades and without a breakthrough in therapy this trend is expected to continue $(1,2)$. The incidence of pancreatic cancer is almost equal to its mortality rate and the estimated 5-year survival rate is only $5 \%(1,3)$.

Nowadays the only potential curative therapy of this disease is radical surgical resection, which can help to increase the 5year survival rate up to $25 \%$ (4). The standard surgical method for curative resection of tumours arising in the head of the pancreas is pylorus-preserving pancreaticoduodenectomy (PPPD) (5). However, the resectability of pancreatic cancer is limited by vessel infiltration. In the presence of tumour ingrowth into adjacent arteries, surgical resection is not generally recommended due to high postoperative morbidity and mortality $(4,6)$. However, tumours infiltrating venous structures [such as the superior mesenteric vein (SMV), and portal vein $(\mathrm{PV})$ ] can be safely resected together with involved part of the vein, resulting in morbidity and mortality comparable with standard PPPD $(4,7)$.

Resection of either PV or SMV during PPPD requires a suitable reconstruction of the involved vessel. In cases when only a tangential resection or short segmental resection is performed, the vein can be reconstructed by venorrhaphy, patch plasty or primary anastomosis. When a longer venous 
segment has to be removed and a tension-free anastomosis cannot be achieved even after the mobilization of abdominal organs, then a reconstruction with interposition graft is required $(5,8)$. For this, autologous venous grafts are used in most cases utilizing e.g. the left renal vein, internal jugular vein, great saphenous vein or superficial femoral vein. Synthetic polytetrafluorethylene (PTFE) grafts are rarely used for PV reconstruction as they are suspected of increasing the rate of postoperative thrombosis and infection (9). Nevertheless, the most commonly used technique using autologous venous grafts still has some disadvantages. These include prolonged operation time, poorly fitting diameter of the graft which has to be adapted, a risk of complications at the site of harvesting, and harvesting itself, which may require an additional incision (10).

Considering the disadvantages of autologous grafts, a reconstruction using allogeneic cadaveric grafts might be a fast and elegant method for reducing postoperative morbidity. Since portal and caval venous systems vary by their perfusion pressure or by vessel capacitance $(11,12)$, we hypothesised that allografts originating from the PV would be ideal for PV reconstruction. However, PV allografts are less available compared to easily accessible allografts from the caval system such as the inferior vena cava (IVC) or iliac veins. The reason for this is the harvesting of PV together with the liver for transplantation purposes. The use of allogeneic venous grafts from organ donors for PV reconstruction has been described in only few studies; in all these cases, grafts from the iliac or femoral vein from the caval venous system were used (13-15). Moreover, to our knowledge, no study examining potential differences between grafts originating from the portal system and the caval system have been reported. Due to the mentioned physiological differences between portal and caval venous systems, a comparison of graft types is very important for safe application and wide use of this technique in clinical medicine.

The reconstruction of PV using venous graft is associated with certain risk of postoperative thrombosis (16). Factors such as composition of the blood, changes of haemodynamics and changes of the vessel wall can trigger thrombosis (17). A non-uniform blood flow and inadequate shear stress stimulation of the vascular wall can cause changes observed at the morphological as well as physiological level (18). Moreover, haemodynamics associated with stagnation or lowvelocity zones or areas with a sudden increase in shear stress, such as vascular stenoses, have a propensity for thrombosis (19). Thus, in order to provide an alternative insight into the problem of portal system haemodynamics and to assess its role in the outcome of PV reconstruction, computer simulations of blood flow in pre- and postoperative PV models were carried out. To our knowledge this is the first time this method has been used to evaluate the risk of thrombosis in reconstructed PV.
The aim of this study was to compare the characteristics of cadaveric venous grafts from PV and IVC in order to evaluate their properties and functionality in a reconstructed portal system to verify the suitability of their utilization. For better intelligibility and because of comparison with clinical medicine the term IVC is used for pig caudal vena cava throughout the article.

\section{Materials and Methods}

Animal experiment legislation. This study was conducted under the authority of the Ministry of Agriculture of the Czech Republic. All the procedures with the use of animals were approved by the Commission for Work with Experimental Animals at the Charles University, Faculty of Medicine in Pilsen, and the whole study was conducted in accordance with the law of the Czech Republic, which is compatible with the legislation of the European Union (number of approval - 4891/2015-MZE-17214).

Experimental animals and testing of immunocompatibility. For the in vivo model of PPPD, Prestice black pied pig was used. All the animals were healthy, about 3-4 months old, with body weight between 24 and $36 \mathrm{~kg}$. Both males and females were included. There were two recipient groups (PV and IVC) and one group of donors. Animals in the PV group received a PV graft $(n=13)$. Animals in the IVC group received a graft of supra-hepatic part of $\operatorname{IVC}(\mathrm{n}=13)$.

One PV and one IVC graft were harvested from each of the donor pigs $(\mathrm{n}=13)$. To avoid incompatibility among individual donors and recipients, a standard blood cross-matching test was performed prior to surgery.

Premedication and general anaesthesia. All animals were under general anaesthesia during all surgical procedures and under analgosedation during ultrasound examination and blood sample collection. Intramuscular premedication used $10 \mathrm{mg} / \mathrm{kg}$ ketamine (Narkamon; Spofa, a.s., Prague, Czech Republic), $5 \mathrm{mg} / \mathrm{kg}$ azaperon (Stresnil; Jannssen Pharmaceutica NV, Beerse, Belgium) and $1 \mathrm{mg}$ atropine (Atropin Biotika; Hoechst Biotika, Martin, Slovak Republic). General anaesthesia was induced and maintained by intravenous administration of propofol (1\% mixture $5-10 \mathrm{mg} / \mathrm{kg} / \mathrm{h}$ propofol; Fresenius Kabi Norges as, Halden, Norway). Intravenous administration of fentanyl (1-2 $\mu \mathrm{g} / \mathrm{kg} / \mathrm{h}$ Fentanyl Torre; Chiesi cz s.r.o, Prague, Czech Republic) was used for continuous analgesia. Airways were secured by endotracheal intubation and pigs were mechanically ventilated. Physiological functions were monitored throughout the surgery. During the procedure, pigs received infusions and volume substitution when needed: Hartmann's Solution (B.Braun Melsungen AG, Melsungen, Germany) and Plasmalyte (Baxter Healthcare Ltd., Compton, UK). Amoxicillin and clavulanic acid (1.2 g Augmentin per pig; GlaxoSmithKline Slovakia s.r.o., Bratislava, Slovak Republic) were used as antibiotic prophylaxis at the beginning of the procedure; the dose was repeated after 2 hours.

Collection of venous grafts. PV and IVC grafts were harvested under aseptic and antiseptic conditions from the pigs in donor group. Before graft harvesting 20,000 IU of heparin (Heparin - Zentiva, k.s., Prague, Czech Republic) was intravenously administered. 
Collected grafts were then stored in saline with $20000 \mathrm{IU}$ of heparine per litre in fridge with temperature around $8^{\circ} \mathrm{C}$. Finally, the donor was sacrificed by intravenous administration of a potassium chloride solution.

PPPD and transplantation of venous grafts. Firstly, ProPort Plastic Venous Access System with PolyFlow polyurethane catheter (Deltec, Smiths Medical Deutschland GmbH, Grasbrunn, Germany) was implanted and introduced through the jugular vein into the superior vena cava for intravenous premedication, administration of crystalloids and blood sample collection. The surgery of recipients followed a standard protocol for PPPD; there was only a small modification involving preservation of the gallbladder, and therefore the bile duct was divided at the level of common bile duct instead of at the level of the common hepatic duct. This step was taken because of easier creation of biliodigestive anastomosis at the level of common bile duct with larger diameter, thus reducing the risk of postoperative complications after such an extensive procedure as PPPD. Then $100 \mathrm{IU} / \mathrm{kg}$ of heparin was administered intravenously to prevent thrombosis during PV occlusion. The freed and encircled PV was then clamped with two vessel clamps proximally and distally to the liver so as to enable the part of the potentially affected PV to be resected and removed together with the whole specimen (the duodenum, proximal part of jejunum, the head and the neck of the pancreas and the part of the PV).

PV reconstruction. For PV reconstruction, the PV or IVC graft was cut into an appropriate length and two end-to-end hand sewn anastomoses were performed to interpose the graft at the place of resected part of the PV. Suture using 5-0 monofilament polypropylene was used. The length of the resected part of the PV and of the graft used for reconstruction was different for individual animals, simulating the situation in clinical medicine when the length of segment which has to be resected depends on the extent of tumour invasion. However, these were equally distributed between both experimental groups to enable the comparison of the groups.

Perioperative measurement. The diameters of the PV, SMV and lienal vein (LV) were measured with sterile caliper prior to vessel reconstruction. The length of the interposed graft, the diameters of the PV, SMV, LV, interposed graft and both anastomoses were measured right after vessel reconstruction.

Restoration of gastrointestinal continuity. The reconstruction of gastrointestinal tract started with end-to-side pancreaticojejunostomy. About 10 centimetres distally from the pancreaticojejunostomy, the choledochojejunostomy was created end-to-side. The stomach was connected with the jejunum with end-to-side pylorojejunostomy followed by side-to-side jejunojejunostomy to avoid regurgitation of the bile and pancreatic juice into the stomach. Then the laparotomy was closed in anatomical layers, and the animals were extubated and allowed to recover.

Follow-up. The animals were monitored every day for the next 4 weeks, focusing on clinical examination to be able to identify any potential surgical complications. The animals fasted for the first 3 postoperative days after the operation and were re-alimented to standard feed portions during the first postoperative week. Access to water was not restricted. Throughout the first postoperative week, $40 \mathrm{mg}$ of pantoprazole (Nolpaza KRKA Slovensko, s.r.o.,
Bratislava, Slovak Republic), $250 \mathrm{ml}$ of Hartmann's Solution and $250 \mathrm{ml}$ of Glucosa 5\% (B. Braun Melsungen AG, Melsungen, Germany) was daily administered via the ProPort system. From the second postoperative week, only $40 \mathrm{mg}$ of pantoprazole was added into the feed every day for the rest of the experiment.

Biochemical markers. Blood samples were collected before the operation, immediately before PV resection, immediately after PV reconstruction, 2 hours after PV reconstruction, and then on postoperative days $7,14,21$ and 28 . Using these samples, serum aspartate aminotransferase, alanine aminotransferase, $\gamma$ glutamyltransferase, alkaline phosphatase, bilirubin, urea and creatinine levels were analyzed.

Ultrasound examination. All transplanted animals underwent ultrasound examination before operation, right after operation and then on postoperative days 7, 14, 21 and 28. Doppler ultrasonography (Ultrasound Scanner Pro Focus 2202; BK Medical, Herlev, Denmark) was performed to verify and quantify the blood flow in the graft and in the PV system. Diameters of the PV, interposed graft, anastomoses, SMV and LV were measured together with the maximum velocity of blood flow in these locations. On the 28th postoperative day, the PV was harvested together with the interposed graft under general anaesthesia. The pigs were then sacrificed by intravenous administration of potassium chloride solution.

Histology. All the explanted PV specimens with the interposed graft as well as the rest of the grafts not used for PV reconstruction were histologically examined. For comparison of the types of grafts at the end of experiment, only the specimens from surviving animals were used. All the tissue samples were spread and stitched to cork plates to prevent tissue deformation and extensive shrinkage and to preserve the orientation of the specimen. All samples were fixed in $10 \%$ buffered formalin and processed to $3 \mu \mathrm{m}$ paraffin sections. Samples were cut along the plane parallel to the long axis of the original vein. The overall morphology was examined using hematoxylin-eosin and green trichrome stains. Type I and type III collagen was examined in circularly polarized light using picrosirius red staining (Direct Red 80; Sigma Aldrich, Munich, Germany). Elastic fibres were stained with Tanzer's orcein (Bowley Biochemical Inc., Danvers, MA, USA). Vascular smooth muscle cells as well as contractile myofibroblasts in the suture area were visualized using immunohistochemical reaction against alpha-smooth muscle actin (monoclonal mouse anti-human smooth muscle actin, clone 1A4, dilution 1:500; DakoCytomation, Glostrup, Denmark). The presence of thrombi, lymphatic infiltration, formation of neointima, and integrity of the endothelial layer was also analysed.

Stereological analysis. Following the stereological principles, we quantified the area proportion of elastin-, collagen-, and actinpositive vascular smooth muscle cells within the venous wall. This was achieved using the point counting method in Stereologer software (Stereology Resource Center, Inc., Chester, MD, USA). These methods were previously established for quantitative histological assessment of the composition of vascular wall (20-22). For the quantification, we firstly took systematic uniform pictures (Nikon Eclipse Ti, obj. 20×, Nikon Instruments Inc., Melville, NY, USA) of the region of interest of each native vein and placed a point grid over the photographs. Then, we counted the number of points hitting the structures of interest and divided this number with the 
number of points of the grid over the image. The result was the percentage of a proportion of quantified parameter.

Computer simulations. For the assessment of pre- and post-operative portal haemodynamics, vessel reconstructions from 10 experimental animals were selected: five from the PV group (PV-9 to PV-13) and five from the IVC group (IVC-9 to IVC-13). Note that not all animals from the experimental group were included in the computer simulations due to the large amount of data required and the difficulties associated with the preparation of simulation models. All vein models used for the computer simulation (30 in total: 10 preoperative, 10 post-operative, and 10 adapted) were reconstructed on the basis of real vein geometries obtained from perioperative measurements and photographs taken before and after the PV reconstruction. The mathematical description of the hepatopetal portal venous flow with small fluctuations related to cardiac activity was based on incompressible Navier-Stokes equations, with blood modelled as a Newtonian fluid. The flow model was numerically solved together with appropriate initial and boundary conditions (23) by means of the cell-centred finite-volume method (24). Fully developed inlet flows simulated in the SMV and LV were based on velocity values measured by Doppler ultrasonography in one of the animals (IVC-12) and used throughout the haemodynamic study as a reference. In other words, SMV and LV velocities prescribed in the venous models of the other animals were proportional to the values found in the IVC-12 animal. This step was taken with the intent to make correlations between each venous model and also to draw general conclusions independently of the haemodynamics of each animal.

Haemodynamic parameters. Beside the usual visualisation of simulated pre- and post-operative results in the form of time average velocity magnitude (TAVM), our aim was to quantify the shear stimulation of vascular wall and to associate its abnormal magnitude with areas susceptible to thrombosis. For this purpose, we used the haemodynamic parameter known as the time-averaged wall shear stress (TAWSS) to describe the time-averaged viscous stress exerted on the endothelium by streaming blood. Note that both low and high TAWSS values are indicators of disturbed haemodynamics and are often denoted as triggers of morphological changes or a primary cause of endothelial injury $(12,25)$. Another marker of disturbed blood flow, prone to thrombus formation due to enhanced blood cell deposition and aggregation, is the relative residence time (RRT) defined by Himburg et al. (26). Because the calculation of particle residence time according to RRT is limited to the immediate vicinity of vascular walls, it was appropriate to introduce another quantity that is able to circumvent this inconvenience and provide an aggregation indicator for the entire vessel. The principle of the technique is to virtually inject the entire vascular model with an 'ink' of a given initial concentration $\mathrm{C}$ and to monitor its gradual decrease with time as the ink is washed away. The residence time (RTc) value within a certain part of the vessel then corresponds to the time it took the ink concentration to drop below a critical value Ccrit. On this basis, it is easy to identify stagnation or low-velocity zones, where the blood cells would show a tendency to deposit and aggregate, thereby increasing the thrombosis risk.

Statistical analysis. Biochemical parameters and results of computational modelling were analysed with respect to group (factor) and measurement time point (level of repeated measurement) using repeated-measures ANOVA. Since the followup measurements of vessel diameters and blood flow included a substantial amount of missing data, repeated-measures ANOVA was not suitable for these and the following approach was used instead. Firstly, in order to assess overall differences between experimental groups over all measurement points, the measured values of each variable were converted into $\mathrm{z}$-scores for each day, these $\mathrm{z}$-scores were then merged and the difference in $\mathrm{z}$-scores between groups was then tested using Mann-Whitney $U$-test. Secondly, in order to evaluate the time progression of the variables, the variable values were converted into $\mathrm{z}$-scores for all available data points for each pig, the z-scores were merged and their development throughout the time points was analyzed using Kruskal-Wallis ANOVA (looking for significantly non-uniform distribution) and Spearman correlation (looking for consistent increasing or decreasing trends). The time trends were assessed both in the whole sample and in the individual groups. Differences in quantitative histological findings were analyzed using Mann-Whitney $U$ and Wilcoxon signed-rank tests. To determine whether the graft types differed in the change of quantitative histological findings between the native and adapted states, two-way ANOVA with interactions was performed and the significance of the interaction factor was assessed. All reported $p$ values are two-tailed and the level of statistical significance was set at $\alpha=0.05$. Statistical processing and testing were performed using STATISTICA data analysis software system (Version 12; StatSoft, Inc, 2013; www.statsoft.com).

\section{Results}

Postoperative complications. PD with $\mathrm{PV}$ reconstruction using allogeneic venous graft from PV or IVC was performed in 26 animals in total. Nineteen animals survived the whole experiment (eight in the PV group, 11 in the IVC group). Seven animals died prematurely because of perioperative or postoperative complications (five in the PV group, two in the IVC group). Two animals died due to thrombosis of extrahepatic part of PV (one in the PV group immediately after the operation, one in the IVC group on the first postoperative day). The other causes of death were as follows: metabolic failure (two in the PV group on the first and second postoperative days), postoperative bleeding in retroperitoneum (one in the IVC group on the first postoperative day), gastrectasia (one in the PV group on the 18th postoperative day), and pancreatic pseudocyst (one in the PV group on the 12th postoperative day).

$P V$ thrombosis. Altogether the thrombosis of the extrahepatic part of the PV was noted in five cases (four in the PV group, one in the IVC group, $19.2 \%$ in total) but only two cases caused death (mentioned above).

Vessel size and blood flow. The results of parameters of portal system measured with Doppler ultrasonography are presented in Figure 1. Only parameters for which values were significantly different between groups or which showed significant trends are presented. 
A

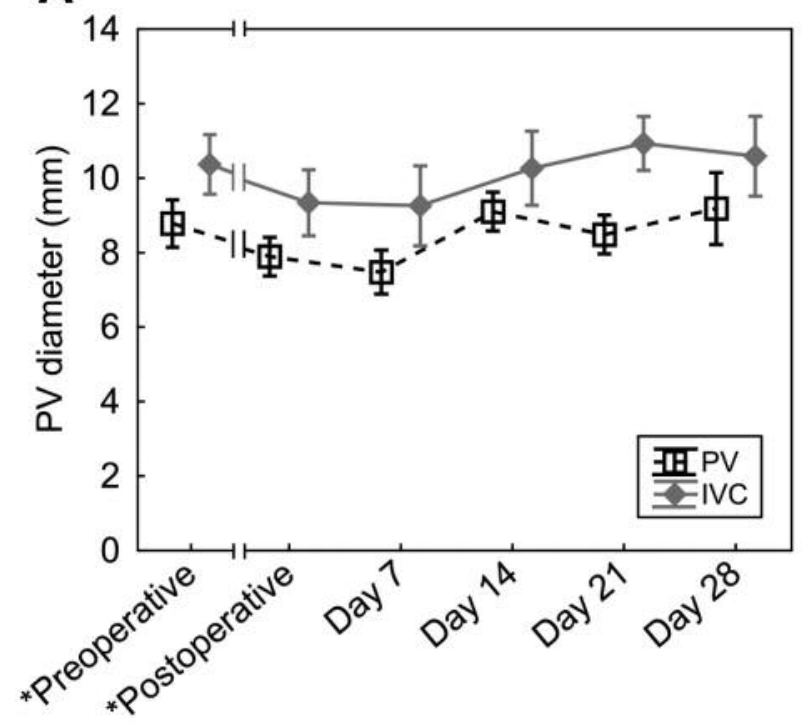

B

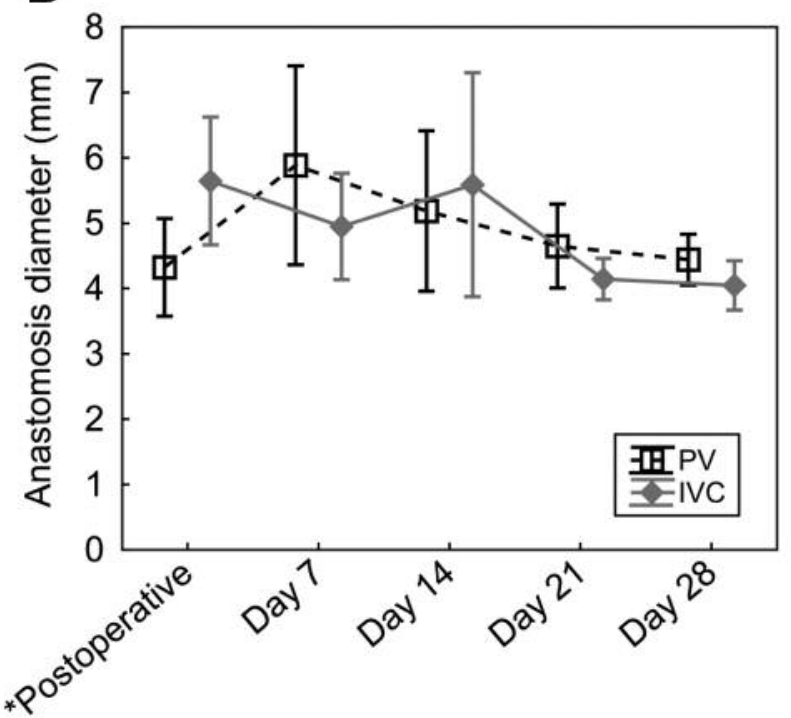

C

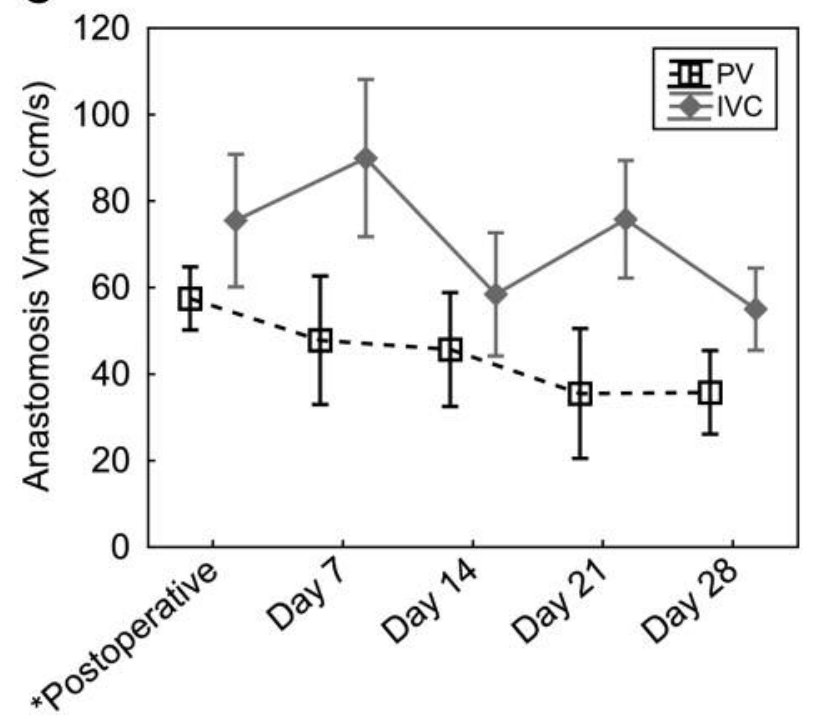

D

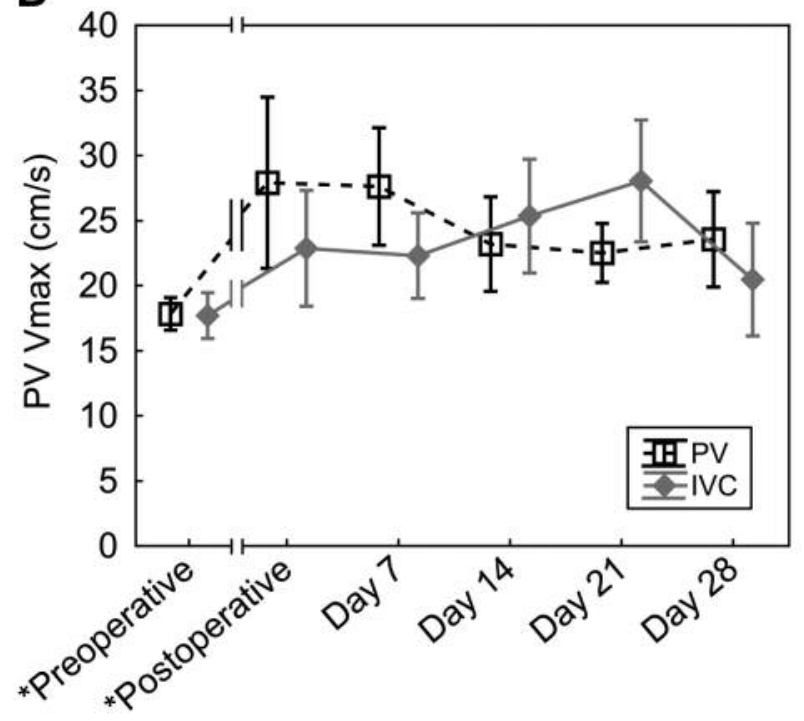

Figure 1. Results of Doppler ultrasonography measurement. A: The diameter of portal vein $(P V)$ was higher in the PV group compared to the inferior vena caval $(I V C)$ group throughout the experiment $(p<0.05)$. The time course was similar for both groups. B: There was a significant trend for narrowing of the anastomosis in the IVC group during the postoperative period $(p<0.05)$. Note the different course in the PV group, where the diameter increased during the first postoperative week. C: The blood flow velocity in the area of anastomosis was higher in the IVC group compared to the PV group $(p<0.05)$. D: Both groups showed a significant increase of blood flow velocity in PV distally to the graft after the graft interposition $(p<0.05)$. Data are the mean \pm SEM. *Immediately.

Graft length. The length of interposed graft was different between individual animals (range $=14$ to $43 \mathrm{~mm}$; mean $=25.3$ $\mathrm{mm}$ ) but it was equally distributed throughout the experimental groups (PV group: range $=20-43 \mathrm{~mm}$; mean $=26.2 \mathrm{~mm}$; IVC group: range $=14-40 \mathrm{~mm}$; mean $=24.4 \mathrm{~mm}$ ). Perioperative measurements revealed PV grafts to be of larger diameter (range $=12-16 \mathrm{~mm}$, mean $=14.2 \mathrm{~mm}$ ) when compared to IVC grafts $($ range $=9-12 \mathrm{~mm}$, mean $11 \mathrm{~mm})(p<0.05)$ (Figure 2).

Biochemical markers. The course of levels of biochemical markers in perioperative and postoperative period are presented in Figure 3. 


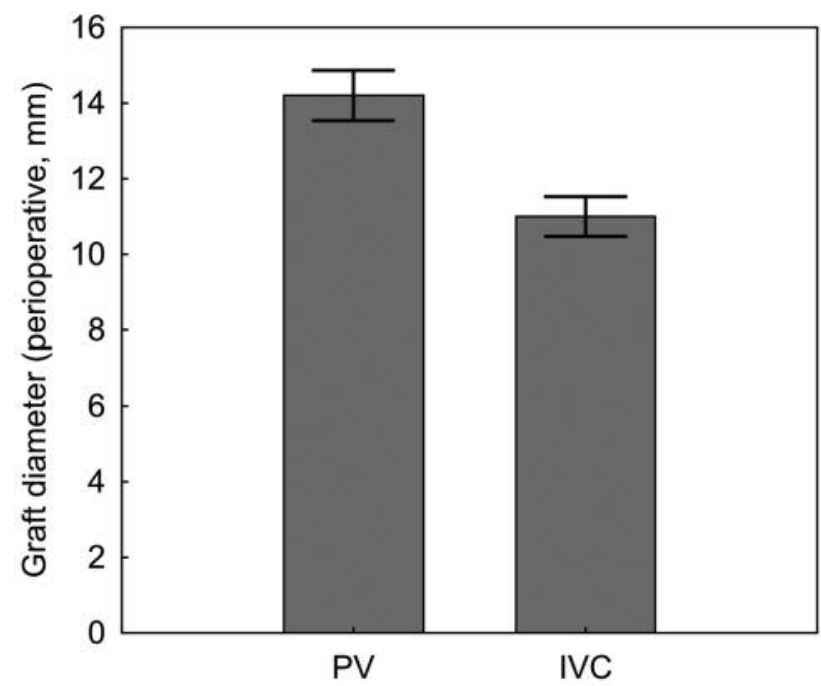

Figure 2. Comparison of the portal vein $(P V)$ and inferior vena caval (IVC) graft diameter measured immediately after its interposition. Data are the mean $\pm S E M$.

Histology. Quantitative histological analysis showed significant differences in the structure of the wall of native PV graft and native IVC graft. The wall of PV grafts before the implantation contained a continuous layer of vascular smooth muscle cells which were absent from the wall of IVC grafts (Figure 4). In agreement with this, the proportion of actin-positive cells was greater in native $\mathrm{PV}$ grafts compared to native IVC grafts ( $p<0.05$; Figure $5 \mathrm{~A})$. On the other hand, native IVC grafts had a greater proportion of collagen I and III fibres compared to the native PV grafts $(p<0.01$; Figure $5 \mathrm{~B})$. The proportion of elastic fibres did not significantly vary between both types of native grafts ( $p=0.35$; Figure $5 \mathrm{C})$. We did not observe any significant histological differences between the two types of implanted grafts after their explantation at the end of the experiment, which demonstrates efficient adaptation of the graft to the implantation site (Figure 5). Related to this, a comparison of the structure of the IVC graft before and after the implantation revealed a significant decrease in the proportion of elastic fibres in transplanted grafts $(p<0.01$; Figure 5C), as well as a greater proportion of contractile cells; nevertheless, the proportion of actin-positive cells was not significantly different (Figure 5A). Transplanted PV grafts lost stratification of smooth muscle layer and showed smaller proportion of actin-positive cells when compared to native PV grafts $(p<0.01$; Figure $5 \mathrm{~A})$. The decrease of the proportion of elastic fibres in PV grafts was not significant ( $p=0.13$; Figure 5C).

Additional histological changes of the implanted grafts that developed during the 4 weeks of remodelling are shown in Figure 6. Both PV and IVC grafts developed thickening of the wall during the remodelling process. In both graft groups, we observed formation of granulation tissue pervaded with smooth-muscle actin-positive contractile cells with formation of micro-vessels, occasionally accompanied by foreign-bodies giant cells. We also detected inflammatory infiltration and proliferation of intima with neointimal hyperplasia. The endothelial layer was preserved to a vastly variable extent, from no endothelial cells detected to a continuous endothelial line. At the end of the experiment, we did not observe a homogeneous layer of contractile cells in either of the groups. The only region-specific stratification was observed at a distance of $600 \mu \mathrm{m}$ from the lumen, where in most of the grafts, a layer of thick elastic fibres was preserved. On the top of this, we identified occlusive thrombi showing signs of organisation and recanalization in several samples. To sum up, for both types of the grafts, the wall was predominantly formed of collagen type I and III, remnants of elastin, and partially organised granulation tissue with interposed contractile cells (smooth-muscle actin-positive cells) (Figure 6).

Computer simulations. Numerical results of computer simulations showing TAVM, TAWSS and RRT are depicted in Figure 7, and show that a change in vessel geometry caused by an interposed graft can have a significant impact on the downstream PV haemodynamic. In all simulated models, the inflow from SMV proved to be dominant compared to the inflow from LV and, thus, played an important role in the resulting blood flow in and after the venous graft. Areas of blood stagnation or recirculation were identified especially in the case of vessels with largediameter grafts, and also in models where the PV line significantly deviated from the direction given by SMV, resulting in a peak velocity skewed towards the wall at the site of the LV inflow (as an example, see results of PV-10 animal in Figure 7A). This peak velocity was heading the area of the graft when longer grafts were used (Figure 7A). In the case of short grafts, the peak velocity was oriented to the area of anastomosis (Figure 7B). The presence of anastomoses was usually accompanied by an increase in TAVM, as is apparent from the model IVC-9 (Figure 7B). According to the 'virtual ink' technique, the highest RTc, which can identify sites with a tendency for blood stagnation, was observed within graft regions, usually near the walls opposite the deflected main stream (at the site opposite the LV inflow) and in large-diameter grafts (Figure 8). Regarding abnormally high shear stress stimulation, which can lead to blood cell damage and trigger thrombosis, very high TAWSS values were particularly noted in areas of both anastomoses and partly downstream from the interposed graft (Figure 7). 
A

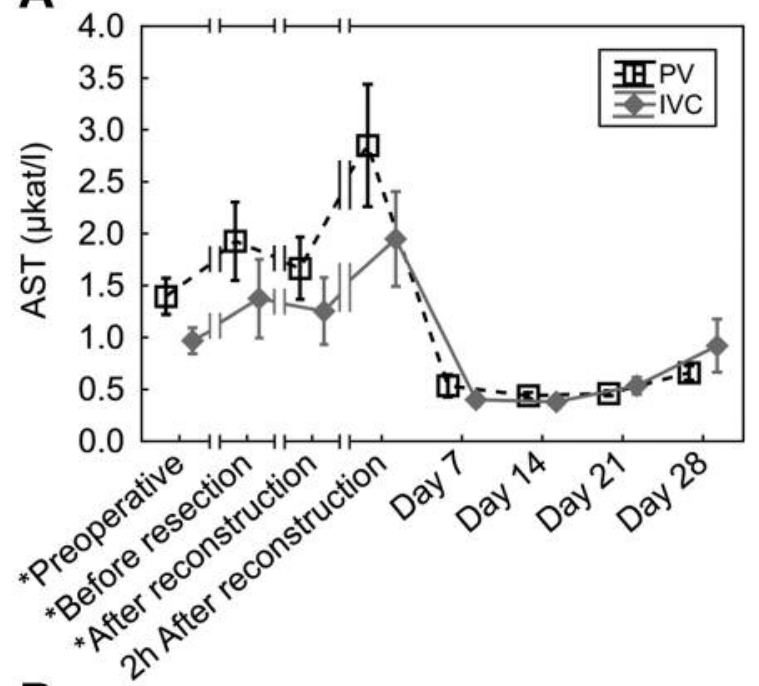

B

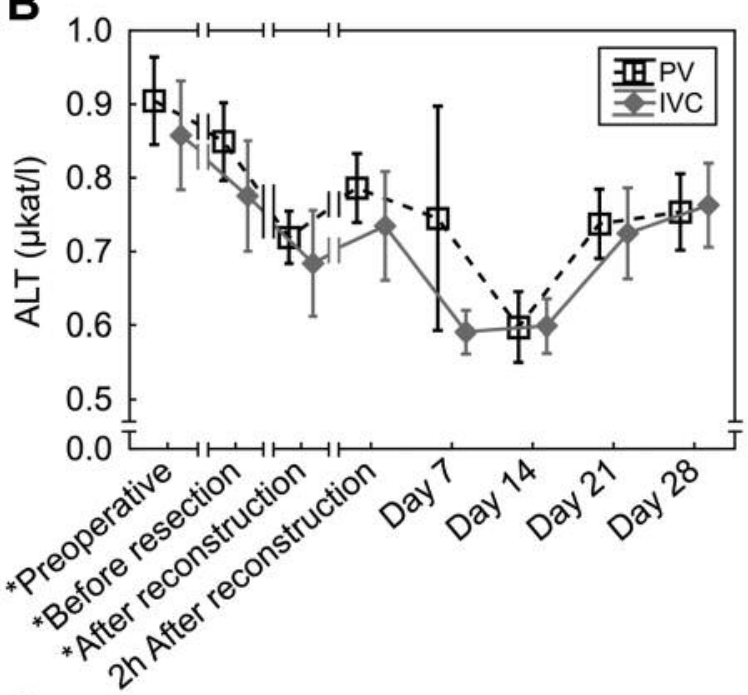

C

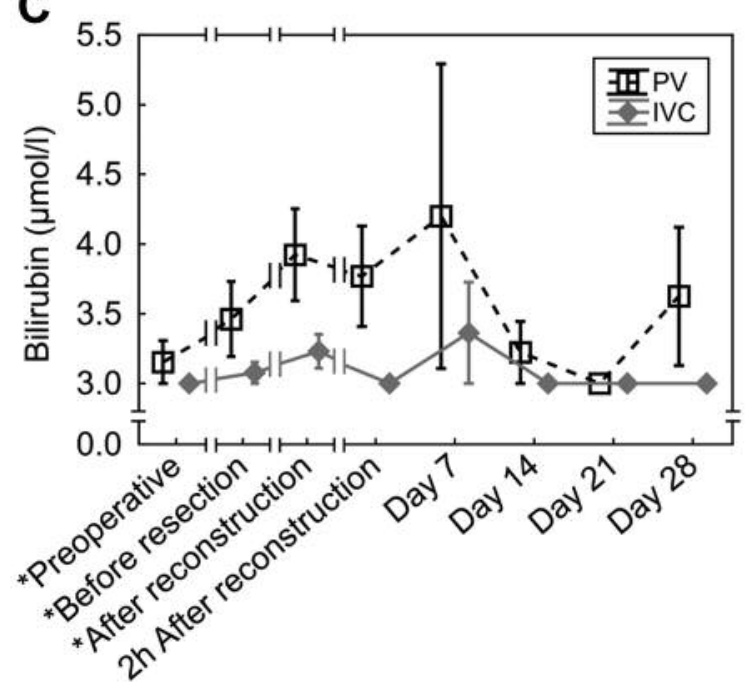

D

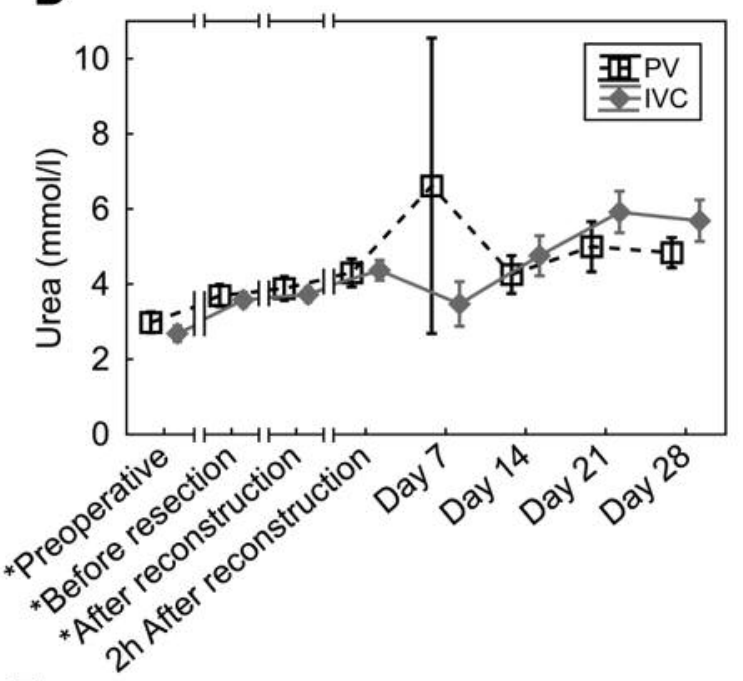

E

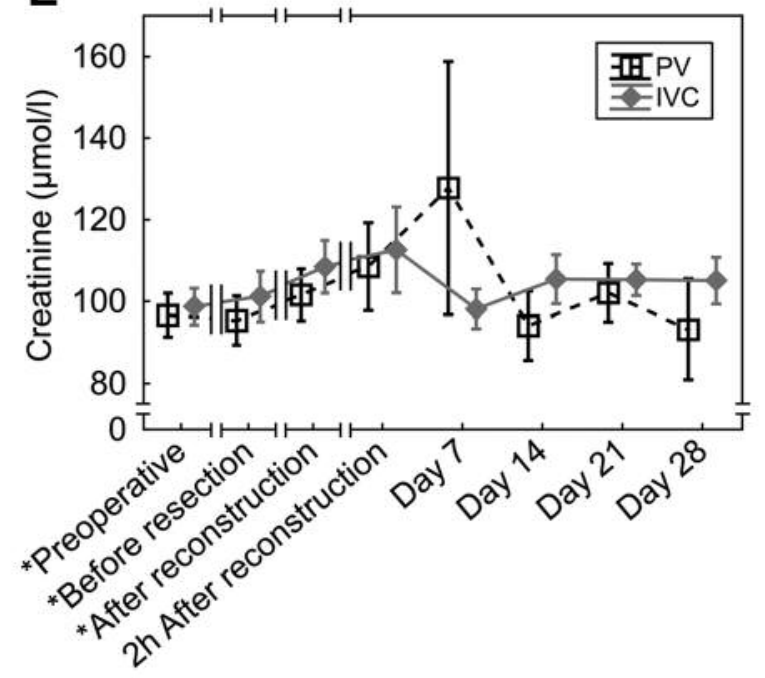

F

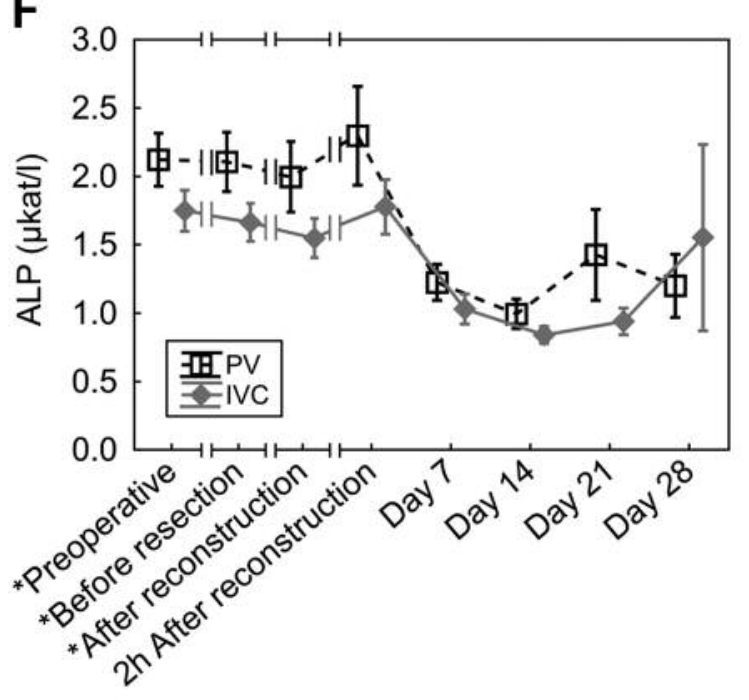

Figure 3. Continued 


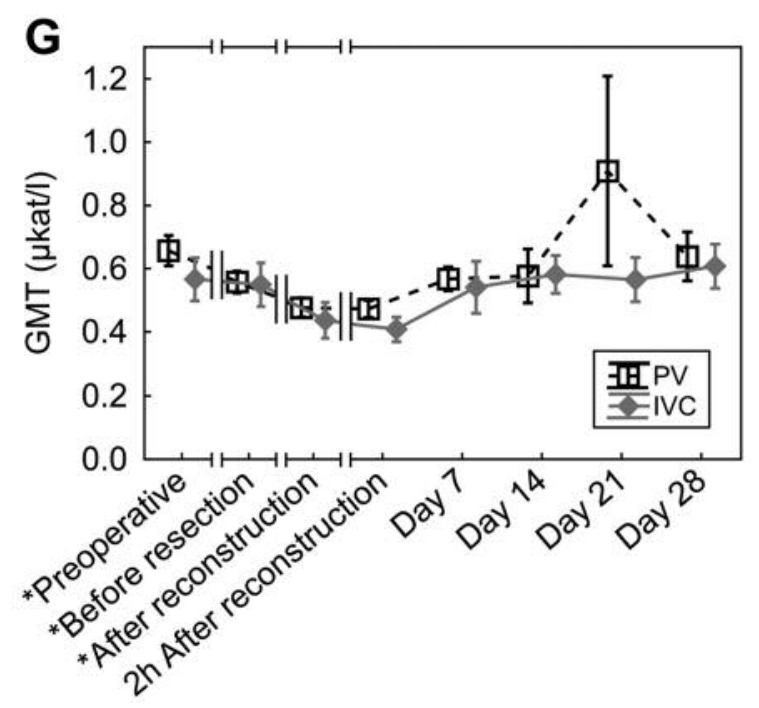

Figure 3. Liver and renal biochemical markers in serum. A: Biochemical analysis revealed elevation of aspartate aminotransferase (AST) concentration in the perioperative period with peak levels at 2 hours after $P V$ reconstruction in both groups. This AST elevation diminished during the postoperative period. B: Serum level of alanine aminotransferase $(A L T)$ slightly decreased in the postoperative period in both groups. $C$ : The serum level of bilirubin was negligibly higher in the PV group perioperatively. D: The level of urea had a tendency to increase but it remained within the normal reference range. E-G: Serum levels of creatinine, alkaline phosphatase (ALP) and $\gamma$-glutamyltransferase (GMT) did not show any impairment of the liver or renal functions during the postoperative period; considering the values of these biochemical markers there were not any significant differences between PV group and IVC group at the individual time points or together for whole period of follow-up. Data are the mean \pm SEM. *Immediately.

Quantification. Quantitative comparison of TAVM, TAWSS, RRT and RTc values of both experimental groups did not reveal any significant differences (data not presented). However, the value of RRT in the PV group increased after graft implantation and decreased in the IVC group. All the aforementioned parameters were also evaluated in relation to the length of the interposed graft to clarify whether the length itself might influence PV haemodynamics, and thus enhance the risk of thrombosis. According to these evaluations, longer grafts were usually associated with more noticeable decrease in TAWSS when comparing postoperative and adapted states of the PV reconstructions.

\section{Discussion}

Due to a low survival rate in patients with pancreatic cancer that is often related to late diagnosis $(1,3)$, there is an urgent need to develop more efficient strategies for tumour resection and suitable reconstruction of involved vessels. We addressed this issue by establishing a pig model of PD followed by PV reconstruction using cadaveric allogeneic grafts. By choosing a large animal model we aimed to imitate a standard clinical PD as much as possible to include all factors which potentially involve the PV reconstruction itself.

We evaluated two different types of cadaveric allogeneic venous grafts originating from PV and IVC in terms of their suitability for PV reconstruction.

There is no reference to usage of allogeneic grafts of PV itself for PV reconstruction after PD, only allogeneic venous grafts of iliac or femoral veins were mentioned (13-15). According to the literature, this is the first report of an established large animal surviving a model of pancreaticoduodenectomy with PV reconstruction.

Histology and physiology. To our knowledge, no study comparing the microscopic structure of the porcine or human PV and IVC has been published. Brown et al. reported a higher collagen content in the wall of IVC compared to the PV in rabbit (12). They also demonstrated correlation between histological findings and functional differences of PV and IVC. PV showed greater capacitance compared to IVC, which allows PV to adapt to the varied needs of the portal circuit. The collagen fibres are probably responsible for greater tension of the IVC wall compared to the more flexible PV (12). In our study, we also observed a greater proportion of collagen in the wall of native IVC compared to that of native PV.

Studies of canine veins showed more smooth muscle cells in the wall of PV compared to other large veins $(27,28)$. In the case of porcine IVC, only a small amount of smooth muscle was observed (29). Our results correlate with these findings. The smooth muscle cells were present in coherent layer in native $\mathrm{PV}$ and they were practically lacking from the wall of native IVC in our study. Brown et al. noted stronger vasoconstriction of PV after noradrenaline administration and more noticeable vasodilatation after removal of calcium compared to IVC. This effect is probably caused by the different wall structure of PV and IVC (higher content of collagen fibres in IVC and more smooth muscle cells in PV) (12).

The ability of greater volume changes of PV according to neurohumoral stimuli might explain the larger diameter of PV grafts immediately after the reconstruction in comparison with IVC grafts. This might potentially be caused by smooth muscle relaxation due to storing of the grafts in saline after harvesting. We also hypothesise that the structure of PV graft wall may cause the dilation of the anastomosis during the first postoperative week which was not observed in the IVC group. However, for verification of this hypothesis, further experimentation would be required.

During the 4-week postoperative period, the PV graft lost stratification of the smooth muscle layer and the smooth muscle actin content significantly decreased. This finding corresponds with the experiment of Yan et al. who studied syngeneic and allogeneic PV transplantation in mice (30). They observed destruction and complete substitution of the 

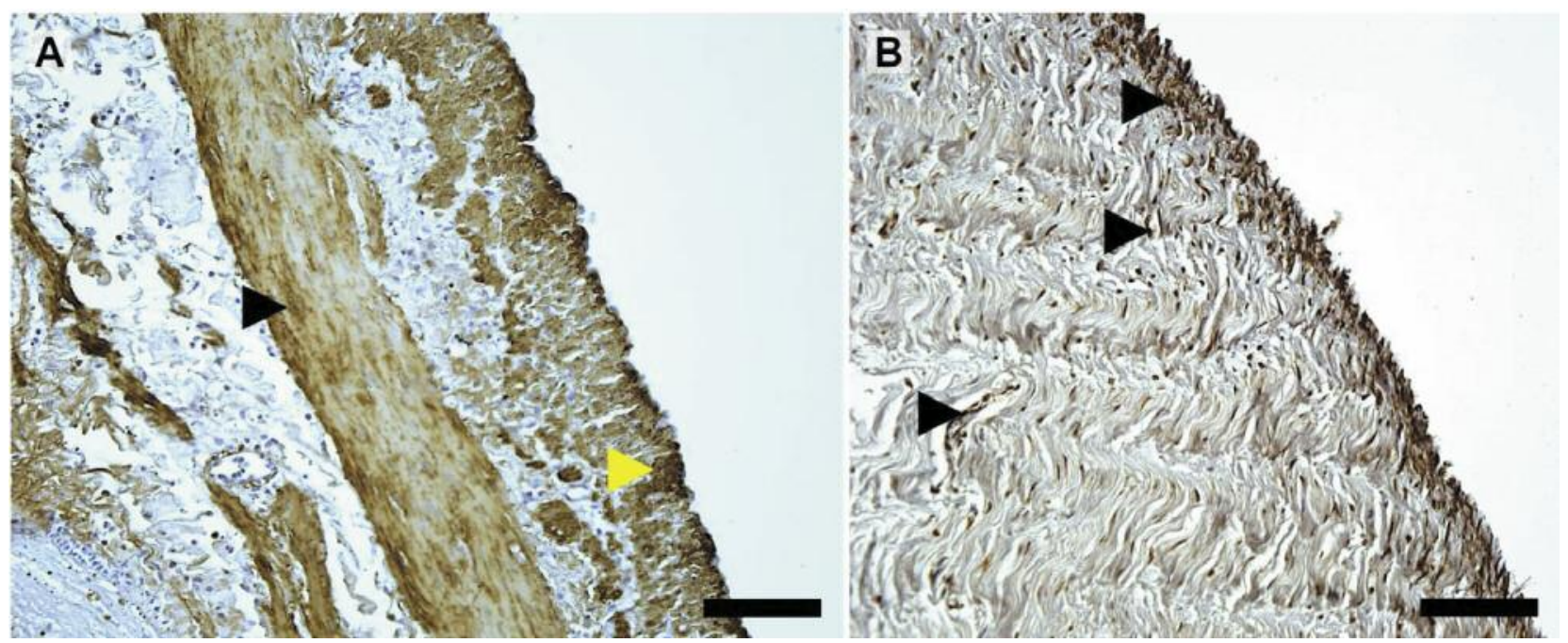

Figure 4. Native portal vein graft (A) and inferior vena caval graft (B) before the implantation. A: Lumen is on the right. In the vein wall, the individual layers were not unambiguously distinguishable. However, there was a continuous layer of smooth muscle actin (SMA)-positive cells in the wall of portal vein graft before implantation (black arrowhead), and smooth muscle actin-positive cells were also present sub-endothelially (yellow arrowhead). B: Lumen is on the right. In the wall of the inferior vena cava graft, the SMA-positive cells did not form a distinct layer (arrowheads). Immunohistochemical staining (monoclonal mouse anti-human SMA), scale bar=100 $\mu \mathrm{m}$.

muscular layer in allogeneic PV graft at 4 weeks after implantation. He explains this change as a result of graft rejection because these changes were not observed in syngeneic grafts nor in the group of mice treated with immunosuppressive agent. Yan et al. observed increase of the wall thickness of the PV allografts between the second and fourth postoperative week (30). Our results also correlate with their findings. We observed thickening of the wall of PV grafts as well as IVC grafts in the 4 weeks after implantation. However, we did not specifically evaluate potential rejection.

The proportion of contractile cells increased slightly in IVC grafts after implantation in our study; however, the proportion of actin-positive cells was not significantly higher. In the case of vein segments grafted into arteries, thickening of the tunica media, growth of smooth muscle, and intimal hyperplasia are described. These changes are caused by mechanical stimuli. According to experiments on dogs, intimal hyperplasia is associated with low flow velocity, and low blood-artery shear stress. The thickening of the tunica media is associated with increased deformation of the vein wall in the circumferential direction (31). Predel et al. in their in vitro study noted proliferation of smooth muscle cells from saphenous vein exposed to cyclic mechanical stretch, whereas such proliferation did not occur in the case of smooth muscle cells from internal mammary artery (32). It is possible that the proliferation of contractile cells in the IVC graft implanted in the PV in this study was triggered by mechanical stimuli, which should be different in caval circulation and portal circulation $(11,12)$.
Another of the observed differences was a significant decrease of the elastin proportion in the IVC grafts. The degradation of collagen and elastin during vein graft adaptation was described in other studies and is caused by mechanical stimuli and oxidative stress (33). We hypothesize that the decrease in the proportion of elastin in the IVC group is a result of changed mechanical stimuli after the transposition of a part of the IVC in the portal system with different haemodynamic properties.

Biochemical markers. The elevation of AST during the perioperative period, with a peak at 2 hours after PV reconstruction might have been caused by general anesthesia in combination with perioperative manipulation and later temporary occlusion of PV during the reconstruction (34). The levels of the other markers remained within the normal reference range. Because there were no significant differences in the observed biochemical markers between the PV and IVC group, it is apparent that the type of graft did not influence liver or renal function.

Computer simulations. To the best of our knowledge, the use of computer simulations has not been previously reported for the study of PV haemodynamics in an experimental animal model. In the past, values of WSS in portal circulation were studied by Wei et al. in cirrhotic patients with PV hypertension (35), using geometrical models of PV prepared according to computed tomographic images. The authors observed lower values of WSS in patients with portal 


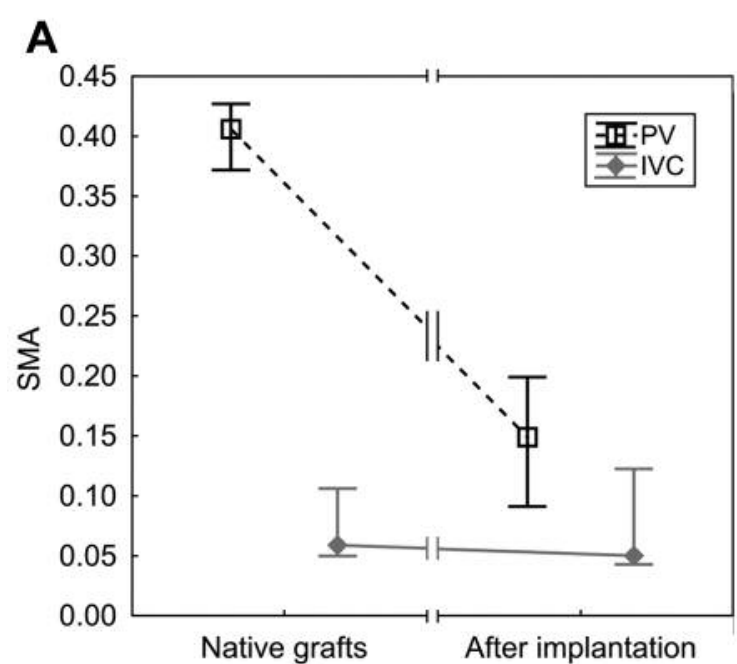

B

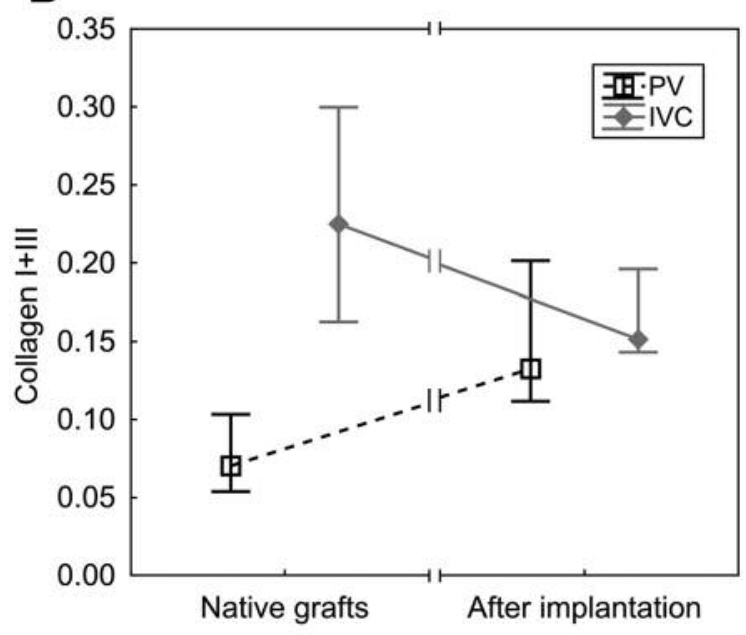

\section{C}

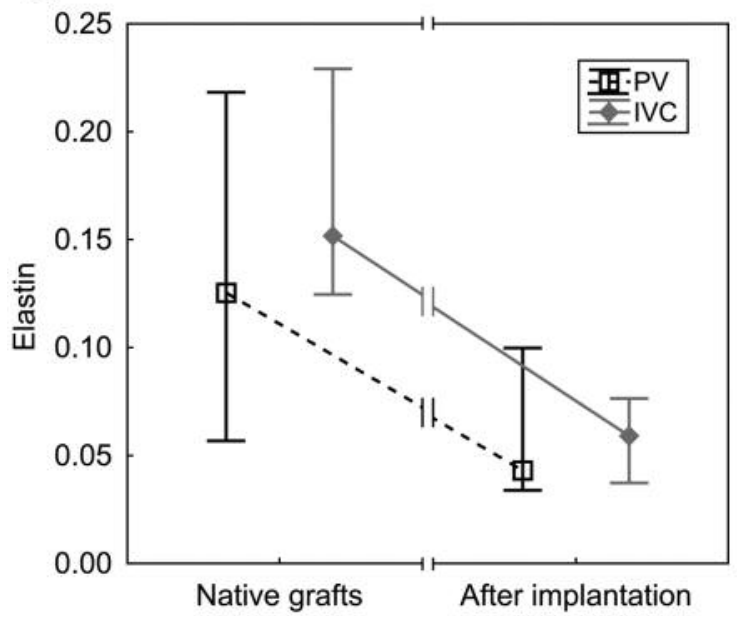

Figure 5. Quantitative analysis of the percentage of staining for smooth muscle actin (SMA) (A), collagen $(B)$ and elastic fibres $(C)$ by area in both types of grafts before and 4 weeks after implantation. See detailed description in the Results section. Data are the mediantinterquartile range. hypertension and noted an influence of the angle between the LV and PV or SMV (34) on the downstream distribution of shear stress. By contrast in our study, these angles did not have a major impact on the resulting PV haemodynamics. This finding can be explained by the fact that in our case, the values of blood flow velocity measured in LV were significantly lower than those in PV and SMV (the peak velocity in LV was usually 10-times lower) as opposed to the values considered in the study by Wei et al. (35), where the difference between the blood flow velocity in LV and PV was minimal.

Influence of anatomic conditions. Furthermore, compared to the aforementioned study and also as a consequence of the dominant SMV flow, our results revealed an influence of the angle between PV and SMV on the downstream haemodynamics (both veins with comparable values of blood flow velocity). In cases when the PV significantly deviated from the direction given by the SMV, the downstream peak velocity had a tendency to shift towards the wall at the site of LV inflow, while the vicinity of the wall opposite the LV inflow was filled with a large recirculation zone.

Influence of graft diameter. Generally speaking, areas of blood stagnation or recirculation were observed to be more common in PVs with interposed grafts of a larger diameter. In our study, the diameter of PV grafts was significantly larger compared to that of IVC grafts immediately after the reconstruction, corresponding to the models simulating the postoperative state. Moreover, the RRT increased in the PV group and decreased in the IVC group after graft implantation. Thus, it can be assumed that the use of PV grafts would probably be associated with a higher risk of thrombosis. This observation seems to be in agreement with the clinical findings, namely, that four out of five thromboses occurred in the PV group.

Influence of graft length. The more noticeable decrease of TAWSS in longer grafts when comparing the postoperative and adapted states might indicate lower risk of thrombosis in these grafts. Usage of longer grafts seems to be reasonable in the case of more noticeable deviation of the PV vein from the direction of SMV. In such a situation, the peak velocity is heading towards the area of the graft, while in the case of a short graft, the peak velocity is oriented to the area of anastomosis, with higher risk of endothelial injury induced by mechanical stress.

Correlation with clinical finding. Other areas susceptible to blood stagnation were identified within graft regions, mainly near the walls opposite the LV and the deflected main stream. This is also in accordance with the clinical findings. The thrombi which developed in reconstructed PVs were 


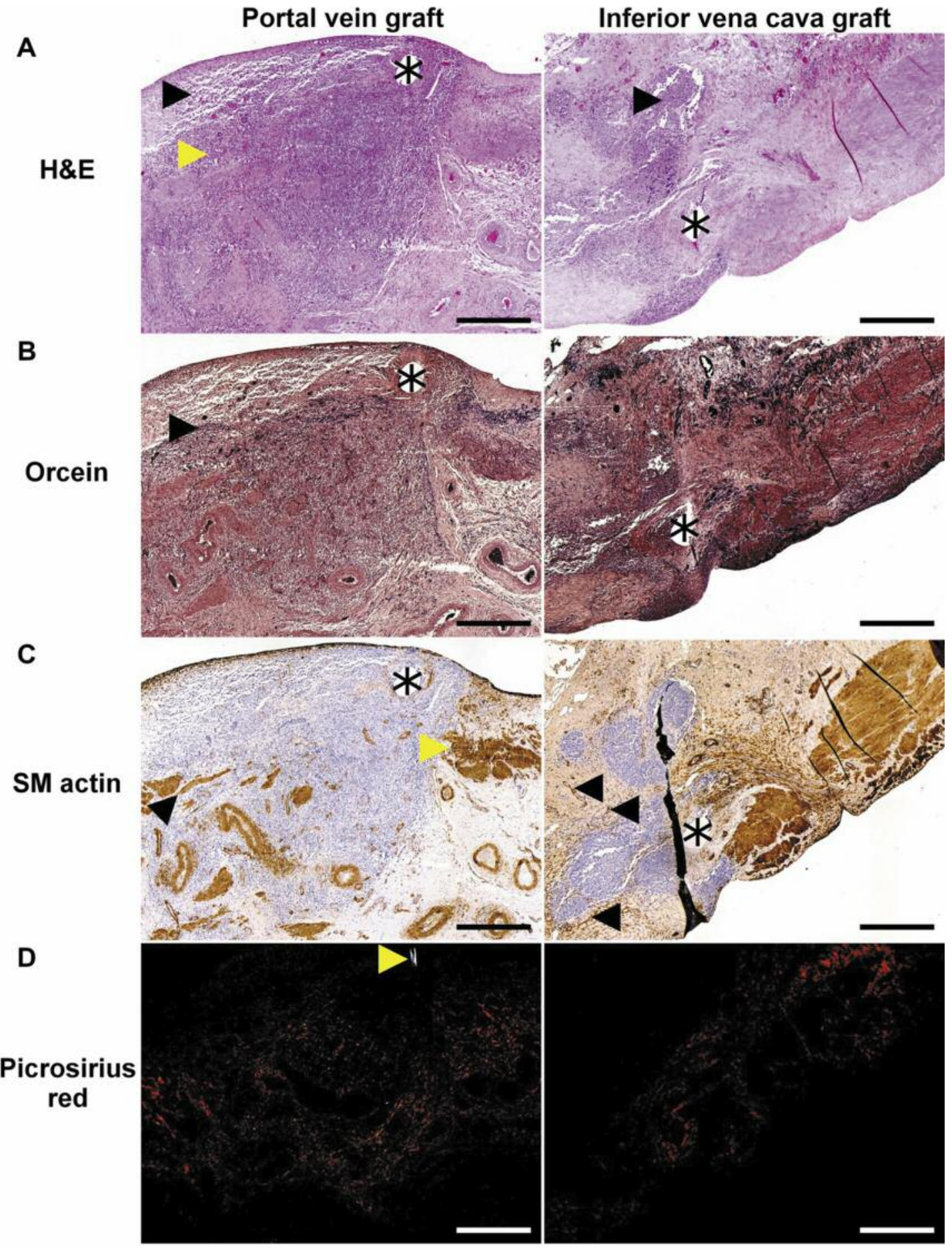

Figure 6. Portal vein (PV) graft and inferior vena caval (IVC) graft 4 weeks after implantation. The graft is on the left and the native vein is on the right of the stitch hole (asterisk). A: Hematoxylin and eosin $(H \& E)$ staining. On the left of the stitch hole (asterisk), proliferation of intima (black arrowhead) and granulation tissue (yellow arrowhead) are visible in the PV graft. Large inflammatory foci (black arrowhead) were visible in the wall of the IVC graft. B: Left: Orcein staining of elastic fibres. Forming of the elastic layer of thick elastic bundles (black arrowhead) is visible at approximately $600 \mu \mathrm{m}$ from the lumen. C: Left: Smooth muscle actin (SMA). Note the remodelling of the original smooth-muscle layer of vena portae (yellow arrowhead) in the PV graft (black arrowhead). In the vein graft, the smooth-muscle actin-positive structures (black arrowheads) were scattered among other components of the graft wall. Large inflammatory foci (black arrowhead) were visible in the wall of the vein graft. D: Under fluorescence microscopy, compare the proportion of collagen type I and III between the specimens. There was no statistically significant difference. Yellow arrowhead in D indicates an artefact within the stitch hole. Scale bar=500 $\mu \mathrm{m}$. 


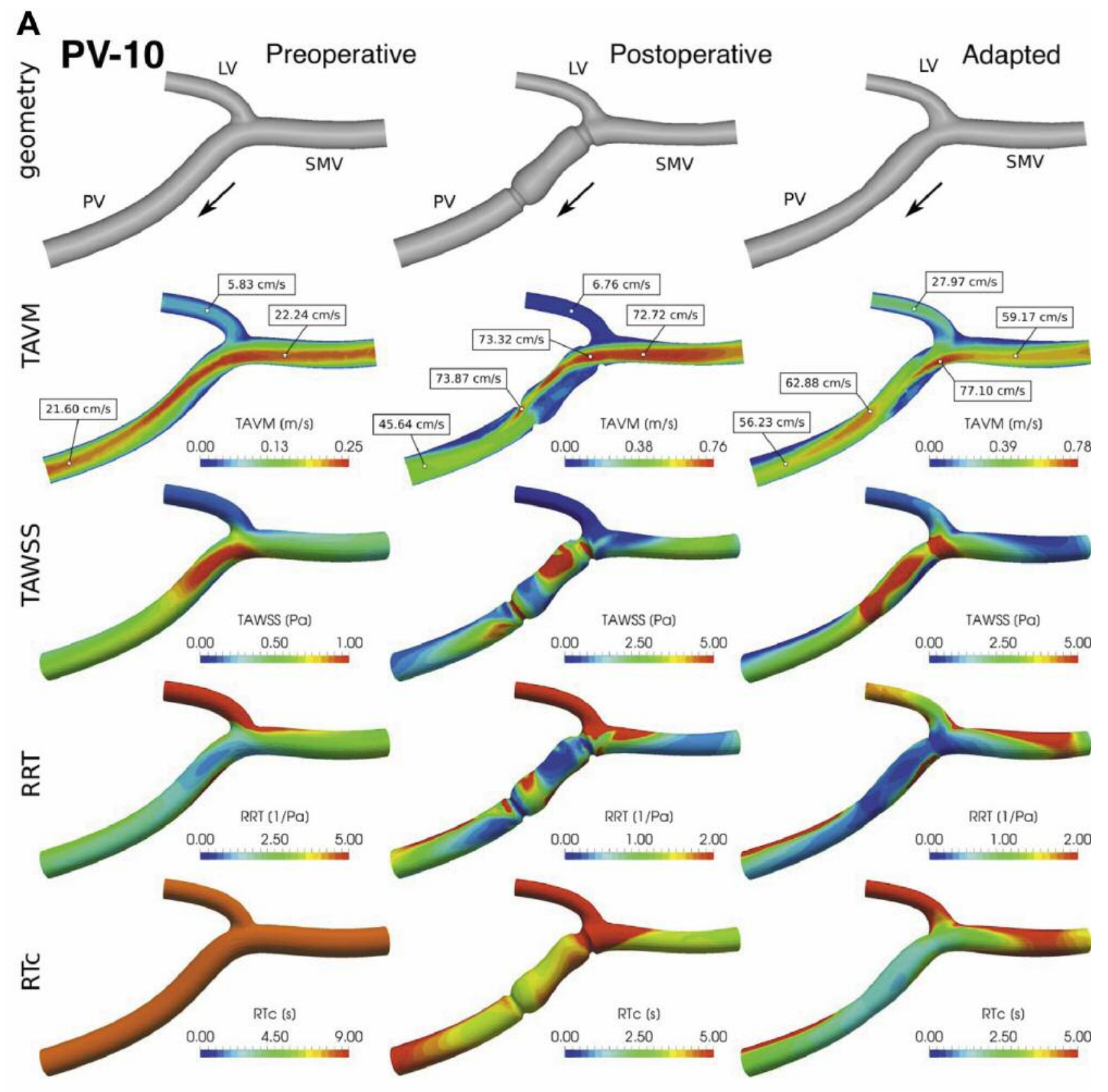

Figure 7. Continued

always located, at least by part of their volume, in the area of the interposed graft.

Possible limitation of computer simulations. The weak point of the haemodynamic evaluation in this experiment may be the planar three-dimensional geometrical model reconstructed on the basis of perioperative measurements and photographs. Computed tomography can provide data for out-of-plane three-dimensional geometrical models in future experiments.
Thrombosis in the PV group. Another reason for a higher incidence of thrombosis in the PV group might be a difference in mechanical properties of the grafts. It was subjectively easier to work with the graft of IVC, which had tougher wall and, as such, it was effortless to perform a technically correct anastomosis with minimal risk of folding of the graft wall that would cause exposure of adhesive molecules of the outer part of vessel wall into the vessel lumen and, consequently, trigger thrombosis (36). 


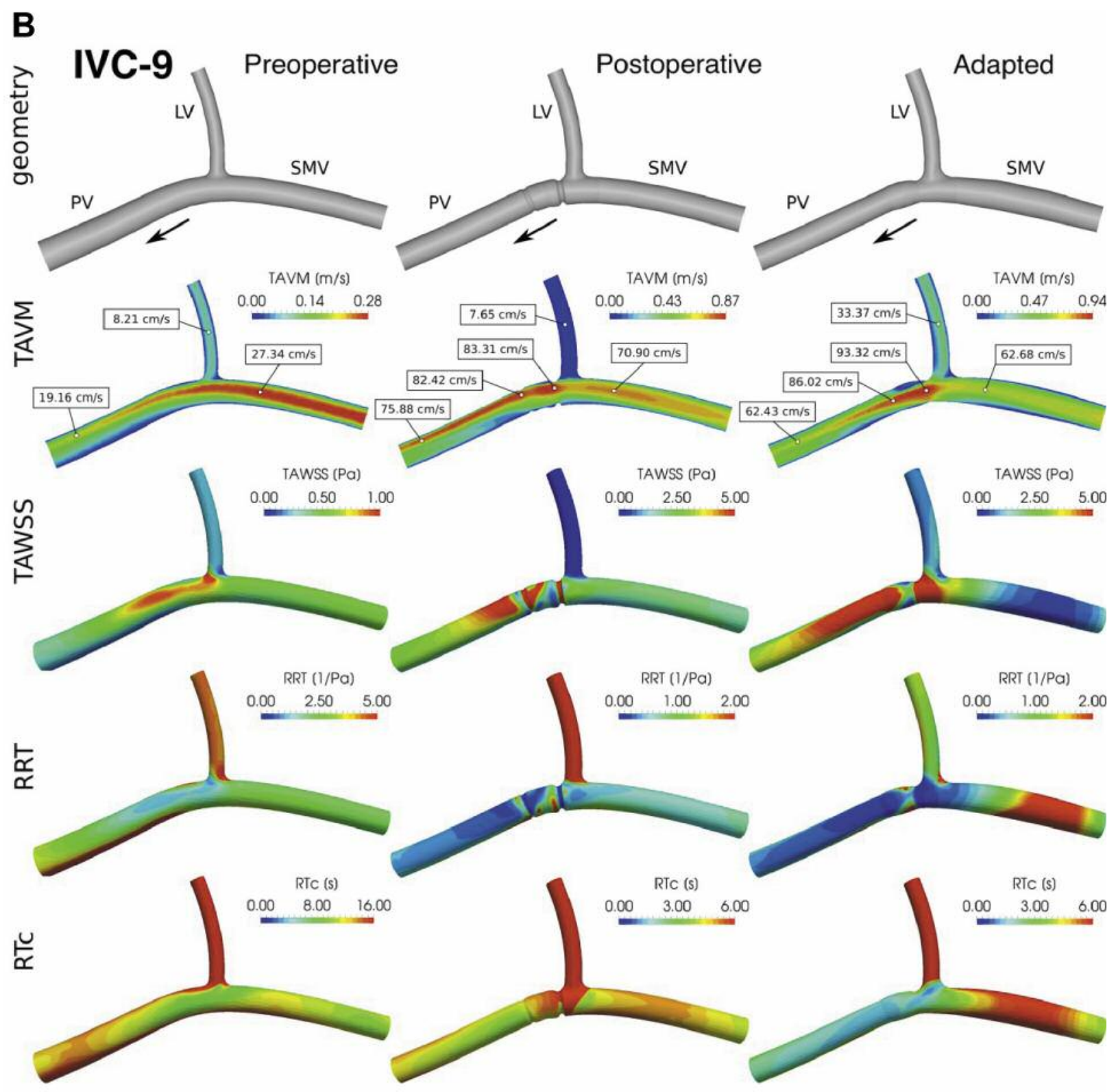

Figure 7. Vessel geometries and computer simulation results for portal vein (PV) reconstruction animal PV-10 from the PV group (A) and IVC-9 from the inferior vena caval group $(B)$. For detailed description please see the text.

$P V$ thrombosis in clinical medicine. The thrombosis rate of $19.2 \%$ in this study concurs with the incidence of thrombosis after PV reconstruction after PD using interposition autologous venous grafts in clinical medicine, which varied between $9.1 \%$ and $36.8 \%(37,38)$.

\section{Conclusion}

To the best of our knowledge, this is the first publication on a large animal experiment studying PV reconstruction after PD using allogeneic venous grafts and the first concerning computer simulation of haemodynamics in reconstructed PV. Histological analysis showed differences in the wall morphology of PV and IVC in the Pig. However, both types of grafts (PV and IVC) developed morphological changes during the 4 weeks after implantation, as they seem to adapt, showing comparable structure at the end of the experiment. Computer simulations based on Doppler ultrasound data demonstrated a significant impact of PV reconstruction on $\mathrm{PV}$ 


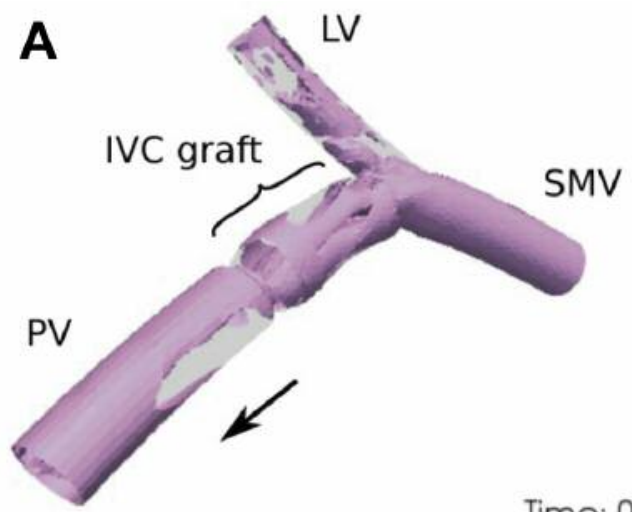

Time: $0.50 \mathrm{~s}$

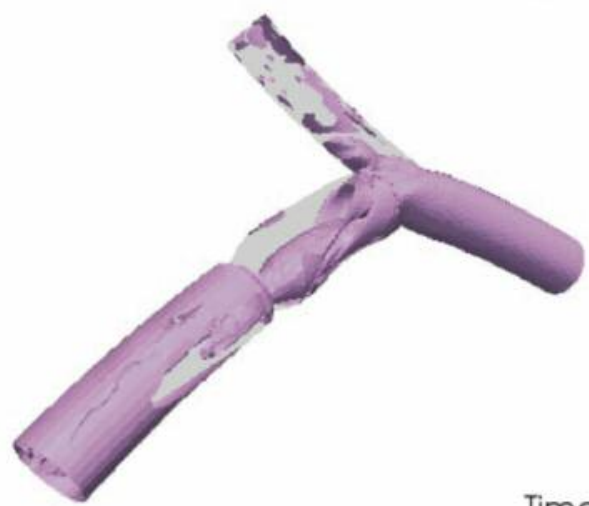

Time: $0.75 \mathrm{~s}$

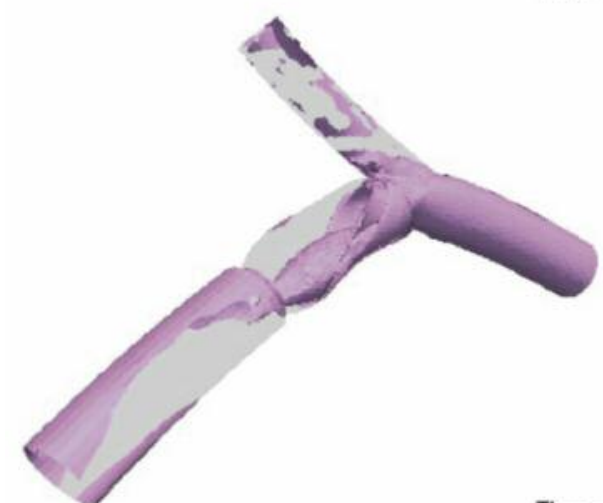

Time: $1.00 \mathrm{~s}$

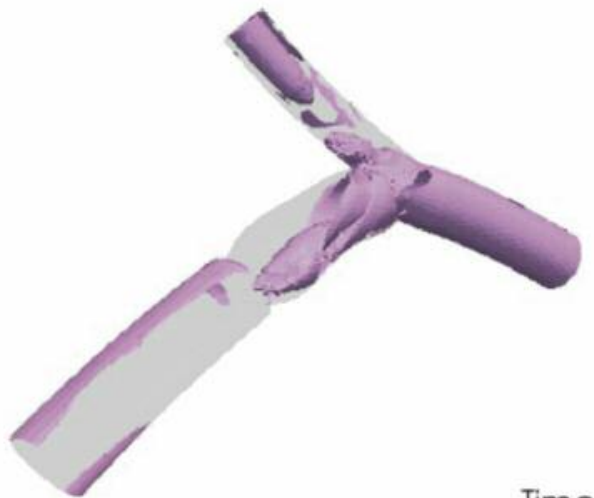

Time: $1.25 \mathrm{~s}$

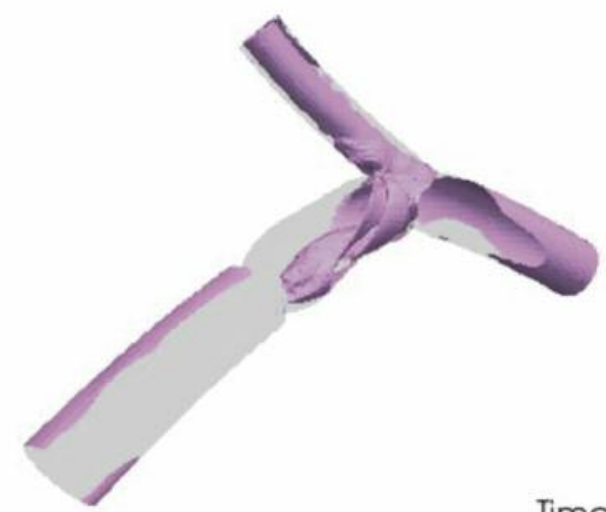

Time: $1.50 \mathrm{~s}$

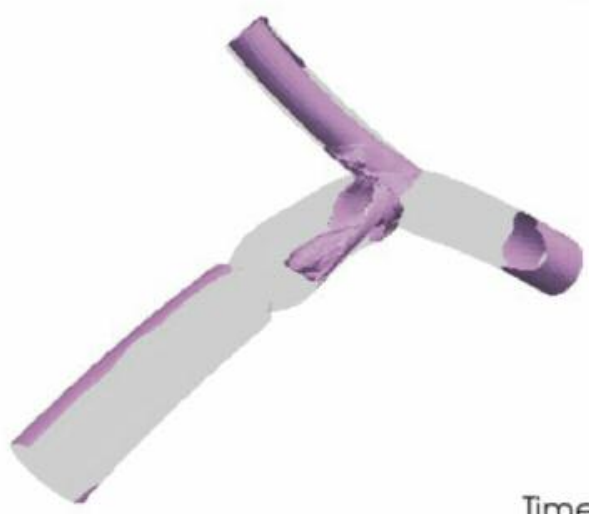

Time: $2.00 \mathrm{~s}$

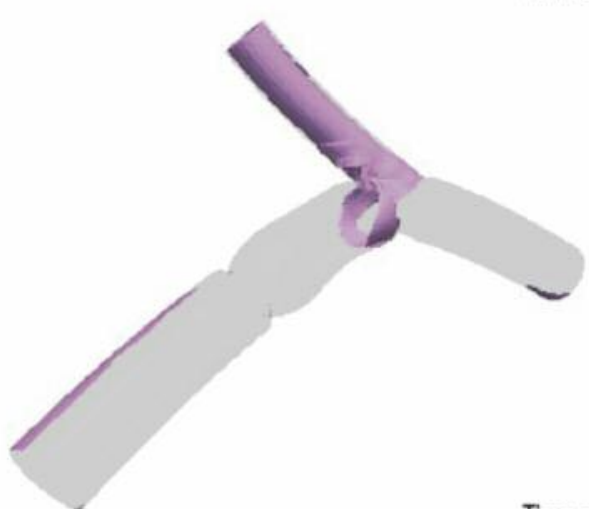

Time: $2.50 \mathrm{~s}$

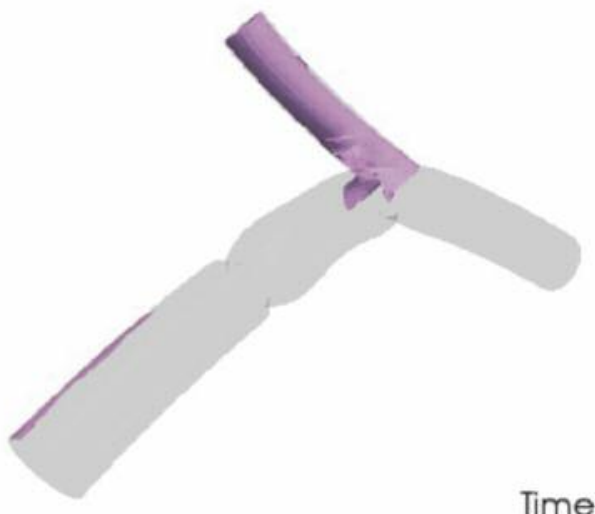

Time: $3.00 \mathrm{~s}$

Figure 8. Continued 


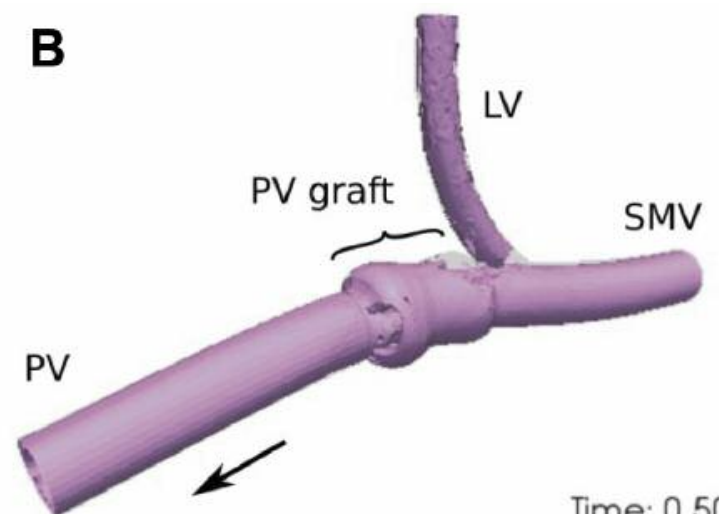

Time: $0.50 \mathrm{~s}$
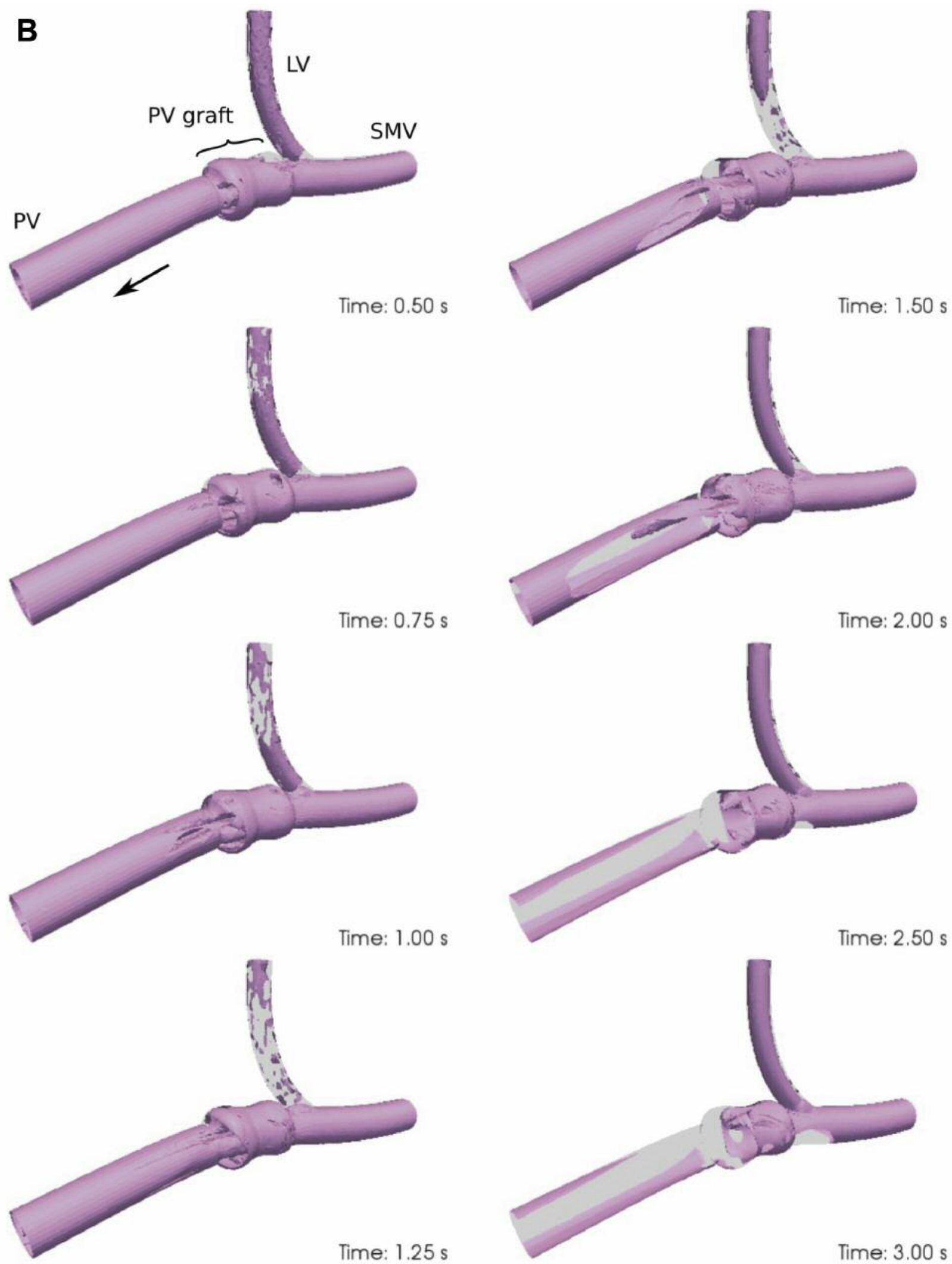

Time: $2.50 \mathrm{~s}$

Time: $1.25 \mathrm{~s}$

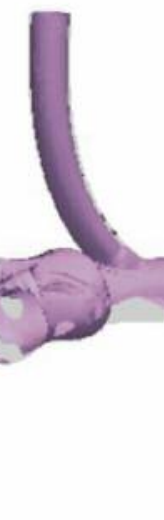

Time: $3.00 \mathrm{~s}$

Figure 8. Continued 


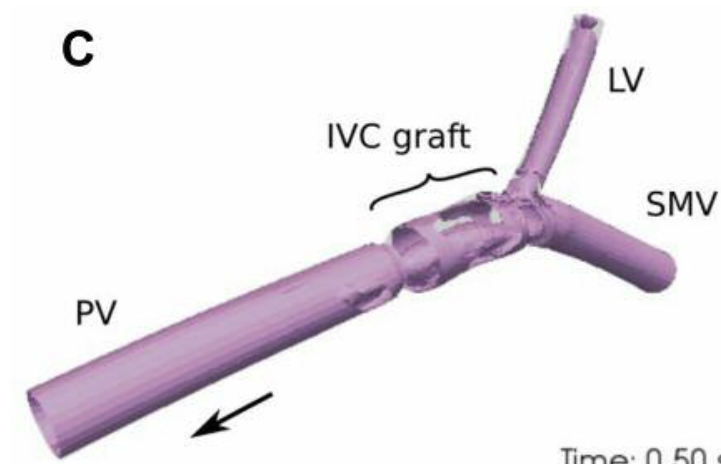

Time: $0.50 \mathrm{~s}$
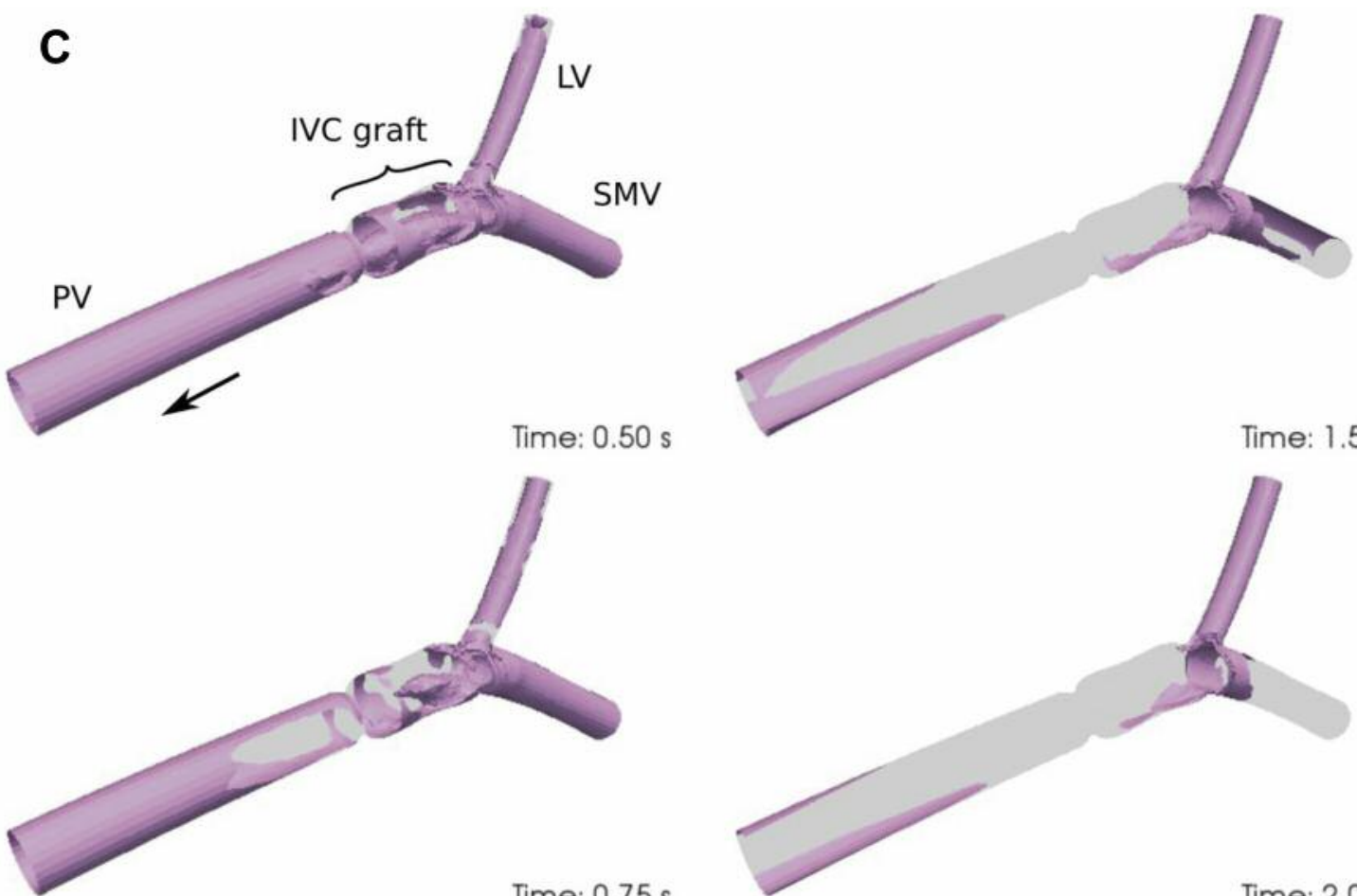

Time: $0.75 \mathrm{~s}$
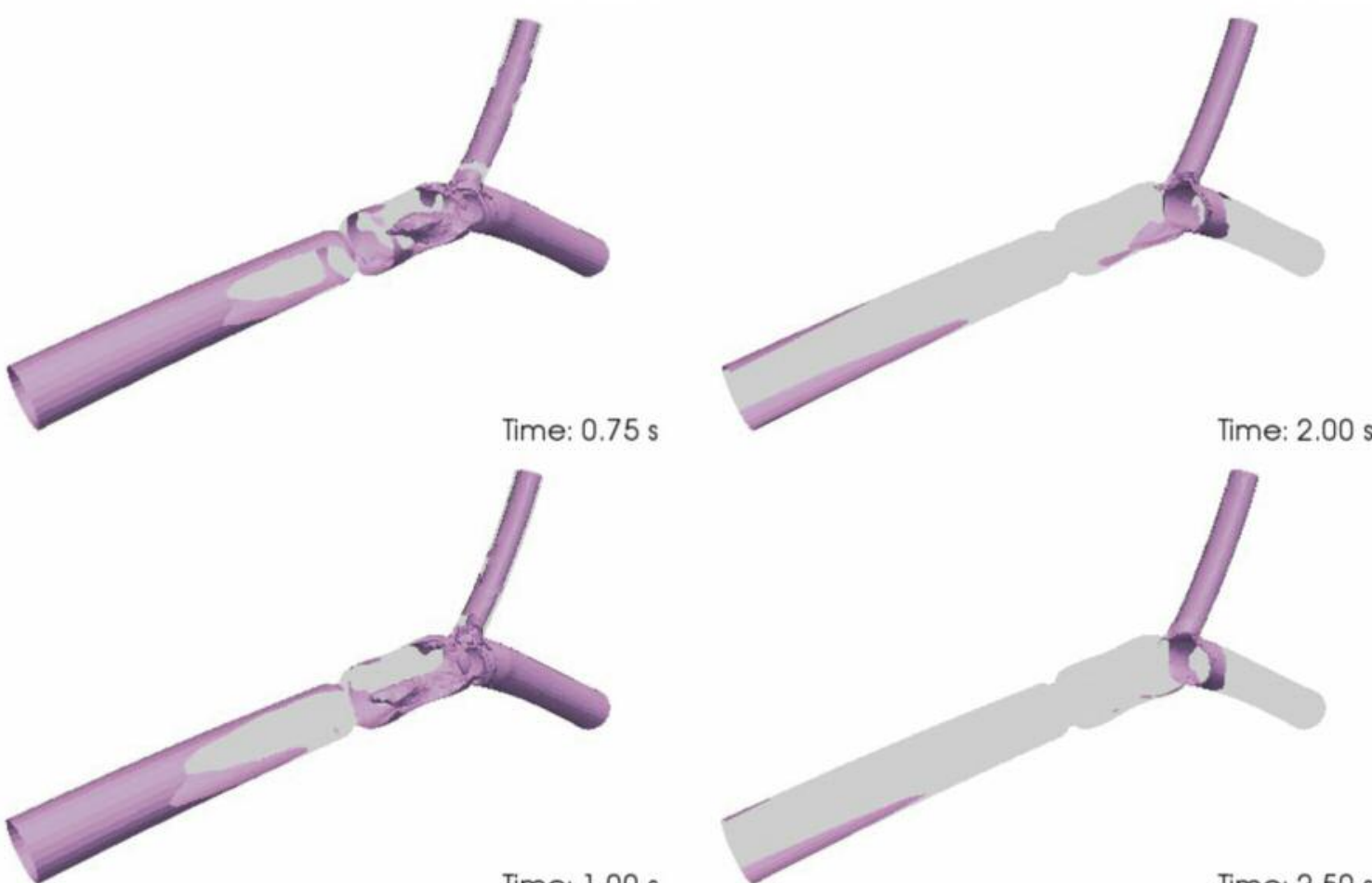

Time: $1.00 \mathrm{~s}$
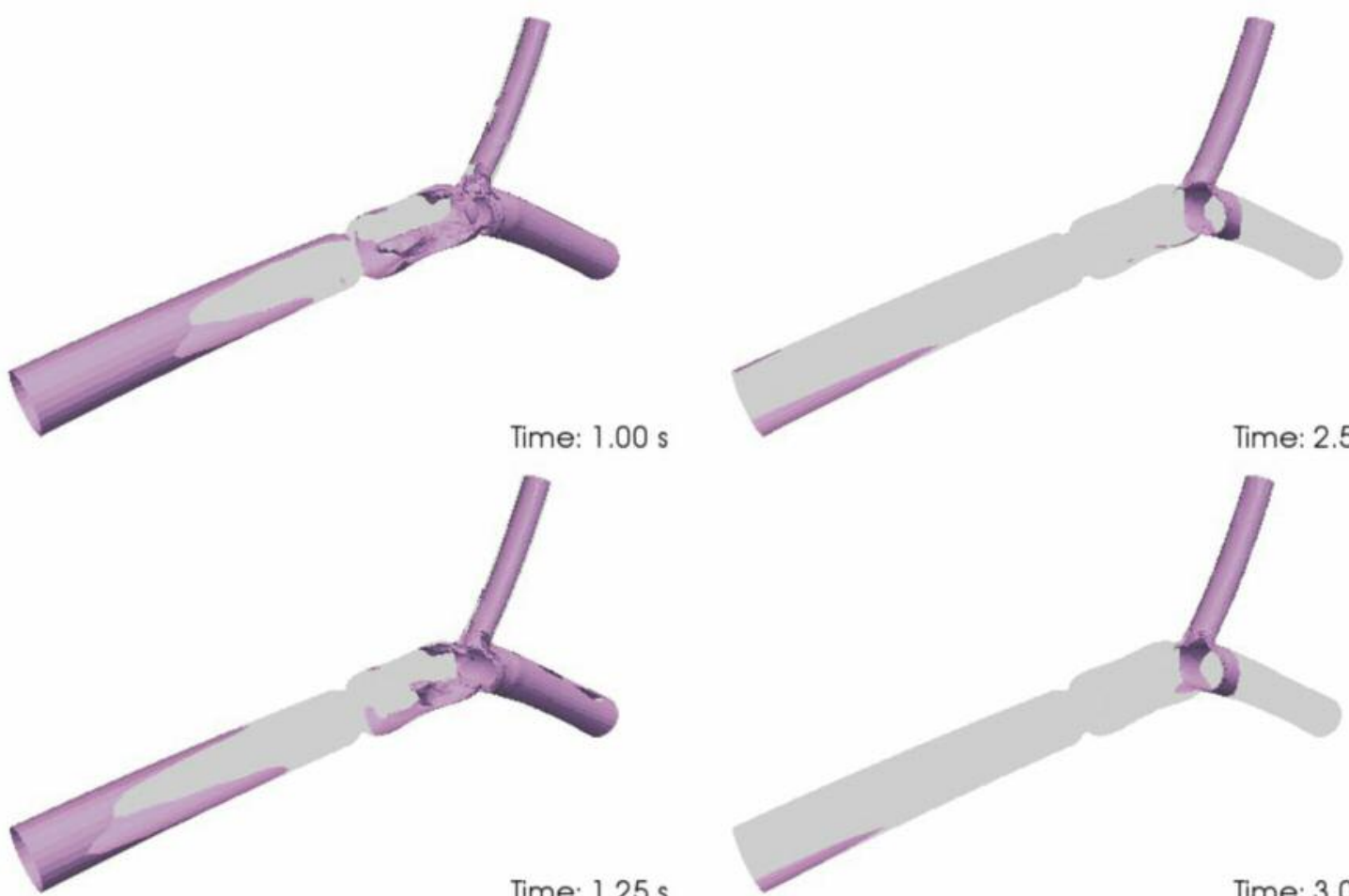

Time: $1.25 \mathrm{~s}$

Time: $2.50 \mathrm{~s}$

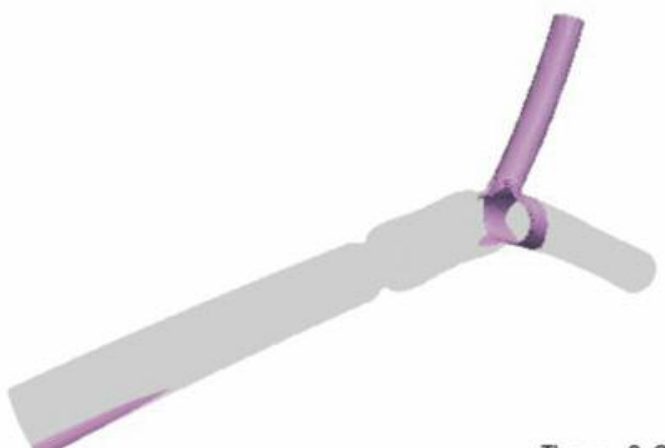

Time: $3.00 \mathrm{~s}$

Figure 8. Computer visualization of blood stagnation in the vessel system obtained by 'virtual ink' technique. Calculation of the residence times (RTc) is shown for three animals: IVC-10 from the inferior vena caval group (A), PV-12 from the portal vein (PV) group (B), and IVC-12 from the inferior vena caval group $(C)$. Areas with the tendency for blood retention (i.e. with the highest RTc values) were observed within graft regions, usually near the walls opposite the deflected main stream (at the opposite site to the lineal vein (LV) inflow) (A). The RTc was higher within grafts with larger diameter $(B)$ compared to grafts with smaller diameter corresponding to the PV diameter $(C)$. Note that higher RTc values observed in the $L V$ were a result of blood flow simulated in this vein (i.e. compared to PV and superior mesenteric vein (SMV) flows, the peak velocity in LV was usually 10-times lower, and thus a longer time was required for the virtual ink to completely leave the vein). 
haemodynamics and demonstrated a higher risk of PV thrombosis in animals with implanted PV graft compared to those with IVC graft. PV thrombosis really occurred more often in the PV group (four cases) compared to the IVC group (one case). This study supports the use of allogeneic venous grafts from the caval system for PV reconstruction in clinical medicine when needed. If possible, individual vessel geometries and appropriate graft lengths should be considered during PV reconstruction with interposition graft.

\section{Conflicts of Interest}

None.

\section{Authors' Contributions}

Richard Palek: Corresponding author, performance of the animal experiment (operation, postoperative follow-up), methodology, data collection, data analysis, data curation, writing: original draft, review. Alena Jonasova: Performance of the computer simulations, data analysis, investigation, methodology. Jan Vimmr: Methodology, supervision, validation. Jachym Rosendorf: performance of the animal experiment (operation, postoperative follow-up), data collection, data analysis. Patrik Mik: Methodology, investigation, performance of the histology examination. Zbynek Tonar: Supervision, validation. Kristyna Bajcurova: Performance and evaluation of the ultrasound examinations, data collection. Petr Hosek: data analysis, data curation, performance of the statistical analysis. Vladimira Moulisova: Investigation, writing, editing. Lada Eberlova: Supervision, validation, investigation. Lenka Haidingerova: Methodology, performance of the animal anaesthesia. Ondrej Brzon: Methodology, performance of the animal anaesthesia. Lukas Bednar: Methodology, performance of the animal experiment (operation, postoperative follow-up). Tomas Kriz: Methodology, performance of the animal experiment (operation, postoperative followup). Martin Dolansky: Methodology, performance of the animal experiment (operation, postoperative follow-up). Vladislav Treska: Supervision, methodology, resources. Vaclav Liska: Corresponding author, validation, supervision, investigation, methodology, funding acquisition, performance of the animal experiment (operation).

\section{Acknowledgements}

The project was supported by grant LO1503 (National Sustainability Program I of the Ministry of Education Youth and Sports of the Czech Republic), UNCE/MED/006 Charles University - Center of clinical and experimental liver surgery and from European Regional Development Fund-Project „Application of Modern Technologies in Medicine and Industry" (No. CZ.02.1.01/0.0/0.0/17_048/0007280).

\section{References}

1 Ilic $\mathrm{M}$ and Ilic I: Epidemiology of pancreatic cancer. World J Gastroenterol 22(44): 9694-9705, 2016. PMID: 27956793. DOI: 10.3748/wjg.v22.i44.9694

2 Mohammed S, Van Buren G and Fischer WE: Pancreatic cancer: Advances in treatment. World J Gastroenterol 20(28): 9354-9360, 2014. PMID: 25071330. DOI: 10.3748/wjg.v20.i28.9354
3 Muñoz AR, Chakravarthy D, Gong J, Halff GA, Ghosh R and Kumar AP: Pancreatic cancer: Current status and Challenges. Curr Pharmacol Rep 3(6): 396-408, 2017. PMID: 29404265. DOI: 10.1007/s40495-017-0112-3

4 D'Haese JG and Werner J: Resektabilität des Pankreaskarzinoms: Neue Kriterien. Radiologe 56(4): 318-324, 2016. DOI: 10.1007/s00117-016-0092-z

5 Bahra M and Neumann U: Surgical techniques for resectable pancreatic cancer. Recent results. Cancer Res 177: 29-38, 2008. PMID: 18084944. DOI: 10.1007/978-3-540-71279-4_4

6. Bockhorn M, Burdelski C, Bogoevski D, Sgourakis G, Yekebas EF and Izbicki JR: Arterial en bloc resection for pancreatic carcinoma. Br J Surg 98(1): 86-92, 2011. PMID: 21136564. DOI: 10.1002/bjs. 7270

7 Ravikumar R, Sabin C, Abu Hilal M, Abu Hilal M, Bramhall S, White S, Wigmore S, Imber CJ and Fusai G: Portal vein resection in borderline resectable pancreatic cancer: a United Kingdom multicenter study. J Am Coll Surg 218(3): 401-411, 2014. DOI: 10.1016/j.jamcollsurg.2013.11.017

8 Yoshitomi H, Kato A, Shimizu H, Ohtsuka M, Furukawa K, Takayashiki T, Kuboki S, Takano S, Okamura D, Suzuki D, Sakai N, Kagawa S and Miyazaki M: Tips and tricks of surgical technique for pancreatic cancer: portal vein resection and reconstruction (with videos). J Hepatobiliary Pancreat Sci 21(9): E69-74, 2014. PMID: 24964060. DOI: 10.1002/jhbp.128

9 Smoot RL, Christein JD and Farnell MB: Durability of portal venous reconstruction following resection during pancreaticoduodenectomy. J Gastrointest Surg 10: 1371-1375, 2006. PMID: 17175456. DOI: 10.1016/j.gassur.2006.09.001

10 Hirono S, Kawai M, Tani M, Okada K, Miyazawa M, Shimizu A, Kitahata $\mathrm{Y}$ and Yamaue H: Indication for the use of an interposed graft during portal vein and/or superior mesenteric vein reconstruction in pancreatic resection based on perioperative outcomes. Langenbecks Arch Surg 399: 461-471, 2014. PMID: 24663295. DOI: $10.1007 / \mathrm{s} 00423-014-1182-\mathrm{x}$

11 Suk KT: Hepatic venous pressure gradient: Clinical use in chronic liver disease. Clin Mol Hepatol 20(1): 6, 2014. PMID: 24757653. DOI: $10.3350 / \mathrm{cmh} .2014 .20 .1 .6$

12 Brown BP and Heistad DD: Capacitance of the rabbit portal vein and inferior vena cava. J Physiol 381: 417-425, 1986. PMID: 3625540. DOI: 10.1113/jphysiol.1986.sp016335

13 Kleive D, Berstad AE, Sahakyan MA, Verbeke CS, Naper C, Haugvik SP, Gladhaug IP, Line PD and Labori KJ: Portal vein reconstruction using primary anastomosis or venous interposition allograft in pancreatic surgery. J Vasc Surg Venous Lymphat Disord 6(1): 6674, 2018. PMID: 29128301. DOI: 10.1016/j.jvsv.2017.09.003

14 Meniconi RL, Ettorre GM, Vennarecci G, Lepiane P, Colasanti M, Laurenzi A, Colace L and Santoro R: Use of cold-stored vein allografts for venous reconstruction during pancreaticoduodenectomy. J Gastrointest Surg 17(7): 1233-1239, 2013. PMID: 23615805. DOI: $10.1007 / \mathrm{s} 11605-013-2201-\mathrm{x}$

15 Zhang XM, Fan H, Kou JT, Zhang XX, Li P, Dai Y and He Q: Resection of portal and/or superior mesenteric vein and reconstruction by using allogeneic veinfor pT3 pancreatic cancer. J Gastroenterol Hepatol 31(8): 1498-1503, 2016. PMID: 26825612. DOI: $10.1111 /$ jgh.13299

16 Gao W, Dai X, Dai C, Jiang K, Wu J, Li Q, Guo F, Chen J, Wei J, Lu $\mathrm{Z}$, Tu M and Miao Y: Comparison of patency rates and clinical impact of different reconstruction methods following portal/superior mesenteric vein resection during pancreatectomy. Pancreatology 16(6): 1113-1123, 2016. PMID: 27707648. DOI: 10.1016/j.pan.2016.09.010 
17 Chung I and Lip GY: Virchow's triad revisited: blood constituents. Pathophysiol Haemost Thromb 33(5-6): 449-454, 2003. PMID: 15692259. DOI: $10.1159 / 000083844$

18 Malek AM, Alper SL and Izumo S: Hemodynamic shear stress and its role in atherosclerosis. JAMA 282(21): 2035-2042, 1999. PMID: 10591386. DOI: 10.1001/jama.282.21.2035

19 Chiu JJ and Chien S: Effects of disturbed flow on vascular endothelium: pathophysiological basis and clinical perspectives. Physiol Rev 91(1): 327-387, 2011. PMID: 21248169. DOI: 10.1152/physrev.00047.2009

20 Eberlova L, Tonar Z, Witter K, Krizkova V, Nedorost L, Korabecna M, Tolinger P, Kocova J, Boudova L, Treska V, Houdek K, Molacek J, Vrzalova J, Pesta M, Topolcan O and Valenta J: Asymptomatic abdominal aortic aneurysms show histological signs of progression: a quantitative histochemical analysis. Pathobiology 80: 11-23, 2013. PMID: 22797551. DOI: 10.1159/000339304

21 Tonar Z, Kubikova T, Prior C, Demjen E, Liška V, Kralickova M and Witter K: Segmental and age differences in the elastin network, collagen, and smooth muscle phenotype in the tunica media of the porcine aorta. Ann Anat 201: 79-90, 2015. PMID: 26232584. DOI: 10.1016/j.aanat.2015.05.005

22 Kubikova T, Kochova P, Brazdil J, Spatenka J, Burkert J, Kralickova M and Tonar Z: The composition and biomechanical properties of human cryopreserved aortas, pulmonary trunks, and aortic and pulmonary cusps. Ann Anat 212: 17-26, 2017. PMID: 28434910. DOI: 10.1016/j.aanat.2017.03.004

23 Vimmr J, Jonasova A and Bublik O: Effects of three geometrical parameters on pulsatile blood flow in complete idealised coronary bypasses. Comput Fluids 69: 147-171, 2012. DOI: 10.1016/ j.compfluid.2012.08.007

24 Vimmr J, Jonasova A and Bublik O: Numerical analysis of nonNewtonian blood flow and wall shear stress in realistic single, double and triple aorto-coronary bypasses. Int J Numer Method Biomed Eng 29(10): 1057-1081, 2013. PMID: 23733715. DOI: 10.1002/cnm. 2560

25 Buchanan JR, Kleinstreuer C and Comer JK: Rheological effects on pulsatile hemodynamics in a stenosed tube. Comput Fluids 29(6): 695-724, 2000. DOI: 10.1016/S0045-7930(99)00019-5

26 Himburg HA, Grzybowski DM, Hazel AL, LaMack JA, Li XM and Friedman MH: Spatial comparison between wall shear stress measures and porcine arterial endothelial permeability. Am J Physiol Heart Circ Physiol 286(5): H1916-H1922, 2004. PMID: 14715506. DOI: 10.1152/ajpheart.00897.2003

27 Brooksby GA and Donald DE: Dynamic changes in splanchnic blood flow and blood volume in dogs during activation of sympathetic nerves. Circ Res 29(3): 227-238, 1971. PMID: 5093284. DOI: 10.1161/01.res.29.3.227

28 Auden RM and Donald DE: Reflex responses of the isolated, in situ portal vein of the dog. J Surg Res 18(1): 35-42, 1975. PMID: 1117713. DOI: $10.1016 / 0022-4804(75) 90139-0$
29 Mattson JM and Zhang Y: Structural and Functional Differences Between Porcine Aorta and Vena Cava. J Biomech Eng 139(7), 2017. PMID: 28303272. DOI: 10.1115/1.4036261

30 Yan S, Zhang Q, Cai M, Yu D, Chen J, Yu P, Zhao Q, Zhou L, Hoffmann $\mathrm{M}$ and Zheng S: A novel model of portal vein transplantation in mice using two-cuff technique. Microsurgery 27(6): 569-574, 2007. PMID: 17705281. DOI: 10.1002/micr.20404

31 Dobrin PB, Littooy FN and Endean ED: Mechanical factors predisposing to intimal hyperplasia and medial thickening in autogenous vein grafts. Surgery 105(3): 393-400, 1989. PMID: 2922677.

32 Predel HG, Yang Z, von Segesser L, Turina M, Bühler FR and Lüscher TF: Implications of pulsatile stretch on growth of saphenous vein and mammary artery smooth muscle. Lancet 340(8824): 878-879, 1992. PMID: 1357299. DOI: 10.1016/01406736(92)93287-w

33 Lu DY, Chen EY, Wong DJ, Yamamoto K, Protack CD, Williams WT, Assi R, Hall MR, Sadaghianloo N and Dardik A: Vein graft adaptation and fistula maturation in the arterial environment. $\mathrm{J}$ Surg Res 188(1): 162-173, 2014. PMID: 24582063. DOI: 10.1016/j.jss.2014.01.042

34 Dabir S, Mohammad-Taheri Z, Parsa T, Abbasi-Nazari M, Radpay B and Radmand G: Effects of propofol versus isoflurane on liver function after open thoracotomy. Asian Cardiovasc Thorac Ann 23(3): 292-298, 2015. PMID: 25227774. DOI: 10.1177/ 0218492314551972

35 Wei W, Pu YS, Wang XK, Jiang A, Zhou R, Li Y, Zhang QJ, Wei YJ, Chen B and Li ZF: Wall shear stress in portal vein of cirrhotic patients with portal hypertension. World J Gastroenterol 23(18): 3279-3286, 2017. PMID: 28566887. DOI: 10.3748/wjg.v23.i18.3279

36 Estevez B and Du X: New Concepts and Mechanisms of Platelet Activation Signaling. Physiology (Bethesda) 32(2): 162-177, 2017. PMID: 28228483. DOI: 10.1152/physiol.00020.2016

37 Krepline AN, Christians KK, Duelge K, Mahmoud A, Ritch P, George B, Erickson BA, Foley WD, Quebbeman EJ, Turaga KK, Johnston FM, Gamblin TC, Evans DB and Tsai S: Patency rates of portal vein/superior mesenteric vein reconstruction after pancreatectomy for pancreatic cancer. J Gastrointest Surg 18(11): 2016-2025, 2014. PMID: 25227638. DOI: 10.1007/s11605-0142635-9

38 Lee DY, Mitchell EL, Jones MA, Landry GJ, Liem TK, Sheppard BC, Billingsley KG and Moneta GL: Techniques and results of portal vein/superior mesenteric vein reconstruction using femoral and saphenous vein during pancreaticoduodenectomy. J Vasc Surg 51(3): 662-666, 2010. PMID: 20080375. DOI: 10.1016/ j.jvs.2009.09.025

Received October 6, 2019

Revised October 23, 2019

Accepted October 29, 2019 